\title{
A monolithic Lagrangian approach for fluid-structure interaction problems
}

\author{
P. B. Ryzhakov · R. Rossi · S. R. Idelsohn · E. Oñate
}

Received: 14 December 2009 / Accepted: 13 July 2010 / Published online: 3 August 2010

(C) Springer-Verlag 2010

\begin{abstract}
Current work presents a monolithic method for the solution of fluid-structure interaction problems involving flexible structures and free-surface flows. The technique presented is based upon the utilization of a Lagrangian description for both the fluid and the structure. A linear displacement-pressure interpolation pair is used for the fluid whereas the structure utilizes a standard displacement-based formulation. A slight fluid compressibility is assumed that allows to relate the mechanical pressure to the local volume variation. The method described features a global pressure condensation which in turn enables the definition of a purely displacement-based linear system of equations. A matrixfree technique is used for the solution of such linear system, leading to an efficient implementation. The result is a robust method which allows dealing with FSI problems involving arbitrary variations in the shape of the fluid domain. The method is completely free of spurious added-mass effects.
\end{abstract}

Keywords Fluid-structure interaction · PFEM ·

Monolithic FSI · Lagrangian fluids · CFD

\section{Introduction}

The simulation of fluid-structure interaction (FSI) problems involving large deformations represents a very active area of research in the field of computational mechanics. Although many different techniques exist to face this kind of prob-

P. B. Ryzhakov $(\varangle) \cdot$ R. Rossi · E. Oñate

International Center for Numerical Methods in Engineering, CIMNE, Barcelona, Spain

e-mail: pryzhakov@cimne.upc.edu

S. R. Idelsohn

ICREA, CIMNE, Barcelona, Spain lems, we will focus here on the interaction between the free-surface flows driven by gravity forces (typically in the low Froude number range) and flexible structures. This area inherits all the difficulties of the free surface flows simulation, and complements them with the ones related to the strong FSI coupling.

The solution of the FSI problem involves the simulation of the fluid and the structural domains and the interaction between them. This can be performed using either partitioned or monolithic methods.

In monolithic methods a single, non-linear, discrete system of equations is written taking into account both the sub-domains (the fluid and the structure) at once. The resulting problem is then solved as a whole, dealing with the non-linearity by some form of Newton iteration. Monolithic methods are robust as they do not introduce any domain splitting error. Their disadvantage is that they lead to large and generally poorly conditioned systems due to different scaling of variables entering the multi-field problem (velocity, displacement, pressure). The poor conditioning has particularly strong impact for the analysis of large scale problems, where direct solvers cannot be used. As a consequence, the use of iterative solvers is necessary and the efficiency of a given monolithic approach often relies on availability of sophisticated preconditioners.

In contrast, partitioned methods rely on the independent solution of the fluid and the structure. Due to the modularity of the approach, existing separate codes can be used for the solution of each subsystem which renders them particularly popular in the FSI community. The coupling is achieved usually by applying fixed point iterations at the fluid-structure interface eventually accelerated to improve their convergence behavior [1]. The fundamental advantage is that the resulting equations systems are smaller and generally better conditioned than the unique monolithic system. 
The problem however shifts to assuring the stability and convergence of the coupling constraint along the interface. Standard segregated Dirichlet-Neumann couplings are efficient, when interaction with stiff structures is considered. Nevertheless they often show instability or poor convergence when applied to the cases where the mass ratio of the subsystems involved is close to one or the domain is completely constrained [2]. This occurs e.g. when modeling the motion of light-weight structures in water.

Our objective in this work is to develop a monolithic method to allow the simulation of FSI systems involving light-weight structures and free surface flows, an area where many partitioned approaches fail. We are convinced that monolithic methods define the optimal choice in the area provided that an efficient strategy for the solution of the resulting linear system of equations is defined. We strive to enable re-utilization of existing structural elements formulations (solids, shells, membranes) within the monolithic algorithm. Therefore, we assume that a standard displacement-based formulation is adopted for the solid domain.

With this goal in mind the first step towards the objective is the choice of the "framework" to be used. Eulerian, Lagrangian or Arbitrary Lagrangian Eulerian (ALE) descrip-
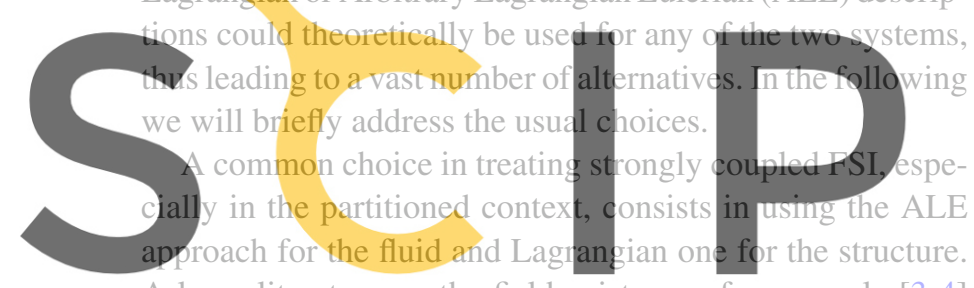

A large literature on the field exists, see for example $[3,4]$ or $[2,5]$. In the ALE approaches the fluid mesh is allowed to

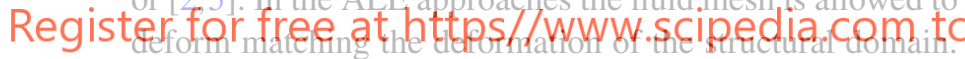

This greatly facilitates the application of the interface boundary conditions and makes ALE-based approaches very accurate. Similarly, space-time approaches [6-8] provide a very accurate framework for the solution of complex FSI problems. References [9] and [10] discuss in detail the time integration scheme, the implications related to the strong coupling algorithm and the techniques used for mesh-moving. An interesting work on the application of the ALE approach for monolithic FSI is presented in [11]. There the formulation is based upon the iso-geometric approach in conjunction with a generalized alpha time integration scheme. The high precision of the method renders it specially attractive for the simulation of complex bio-mechanical problems. Unfortunately, even the most advanced ALE formulations arrive to their limits when the domain shape deformations are large. In such situations, re-meshing is inevitable [12]. This is the case, in particular, for problems involving free-surface gravity flows, which are of interest in the present work. The option of using an ALE-based fluid equipped with adaptive re-meshing and alpha-shape technique for detecting the free surface was explored in [13].
A second option would rely on keeping a standard Eulerian formulation for the fluid and a Lagrangian one for the structure allowing an overlap between the different domains. Such choice allows using standard formulations for each of the subdomains and is used, for example, in [14] and [15]. The FS interface in such setting is found as an intersection between the Lagrangian and the background Eulerian mesh. Complications arise in the application of the interface boundary conditions that describe the interaction, and are generally dealt with via the definition of an additional interface equation, ensuring kinematic compatibility and absence of flux across the interface. A number of methods exist for the interface treatment. These include, among others, penalty, Lagrange multipliers and augmented Lagrangian methods. In Lagrange multipliers and augmented Lagrangian methods the interface equation involves an additional variable (the Lagrange multiplier $\lambda$ for the force at the interface), thus leading to further deterioration of the monolithic system conditioning. It is worth mentioning however that in the context of partitioned approaches, the Lagrange multiplier-based interface equation is successfully employed in some recent works, see for example [12].

The use of an Eulerian description for both the sub-

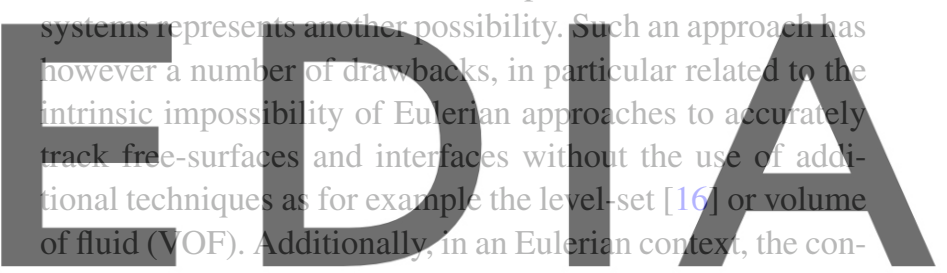

stitutive laws assume a cumbersome form (with the possible exception of simple linear-elastic relations [17]). From the d pomf pad the version without the watermark

also implies a complete re-writing of the structural module, which is usually written in the Lagrangian framework.

The approach we propose in this work uses Lagrangian formulations for both the fluid and the structure. Such an assumption implies the need for frequent re-meshing and results in a method, called "The particle finite element method", which is hybrid between the FEM and a mesh-free techniques. This class of Lagrangian methods was proposed in the late 90s $[18,19]$ and developed further in recent works $[20,21]$. The validation of the method can be found in [22]. The basic philosophy of the PFEM is adopted in the current work. In the context of FSI the use of a Lagrangian fluid together with a Lagrangian structure eliminates the problem of free surface and interface tracking and leads to symmetric system matrices due to absence of the convective term in the momentum equations.

The method we develop in the current work is an extension of PFEM. It is, however, specifically designed for the simulation of the interaction of gravity-driven flows with flexible structures. For such category of problems, Lagrangian methods such as PFEM or Smoothed-Particle 
Hydrodynamics (SPH) have shown once and again their competitiveness with respect to their Eulerian counterparts. Our current proposal originated from an idea that is often used in the SPH community: introducing a certain level of compressibility for the fluid, considerably simplifies the computations in the SPH. We strive to retain some of the advantages of such assumptions while allowing the use of more realistic values for the sound propagation speed. Although classical incompressible ALE techniques are definitely possible in a PFEM context (and were actually used by the same authors in previous works), our aim was to explore here the possibility of taking advantage of the compressibility in devising a strongly coupled monolithic approach.

A first proposal of compressible monolithic FSI based upon the Lagrangian approach for both the fluid and the structure was presented in [21]. The paper assumed a discontinuous pressure thus resulting in an FSI system, written exclusively in terms displacements. The method was proved to be robust and efficient and was successfully used for the simulation of the fluid-membrane interaction in [23]. However, due to the use of discontinuous pressures it is plagued by severe locking problems which hinders its applicability for high values of the bulk modulus.
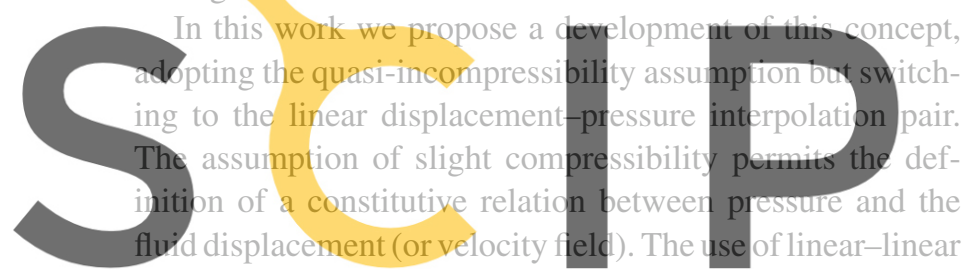

displacement-pressure pair cures to a great extent the locking problem, but precludes elemental presssure condensation.

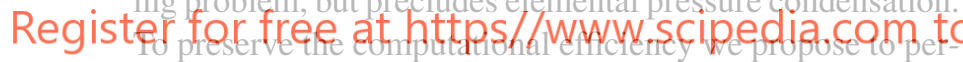
form the pressure condensation at the global level in order to obtain an equivalent linear system written exclusively in terms of displacements. The way such pressure condensation is actually carried out has a strong impact on the computational efficiency and is therefore addressed in detail in this work.

The use of equal-order simplicial elements implies on the other hand a loss of pressure stability as the incompressible limit is approached. A simple pressure stabilization technique, based upon the difference of the consistent and lumped mass matrices is introduced. It is shown that this technique is similar to the Laplacian-type stabilization.

The paper is organized as follows: an updated Lagrangian quasi-incompressible fluid formulation is derived first. A global pressure condensation strategy is then described. Evidence is provided that such approach provides a considerable advantage in comparison to the solution of the original displacement-pressure fluid, even for a single field fluid problem. An efficient implementation, based on the use of a matrix-free iterative solver is described providing an estimate of the savings in terms of floating point operations. The procedure is finally generalized to include the interaction with the structure, which is introduced in the monolithic system by a completely standard FE assembly process.

In our work the variables of interest in the continuum are denoted by lower-case letters; vector quantities are indicated in bold. The corresponding discrete vectors, containing the nodal values which correspond to the finite element implementation, are indicated by adding an over-bar to the corresponding symbols. Bold capital letters are used for matrices.

As an example, the symbol $p$ represents the pressure at a given point of the continuum, while $\bar{p}$ indicates the vector of pressures of all the nodes of the FE mesh.

Time step index is located in bottom-right position. Non linear iteration counter is located in apex position. For example

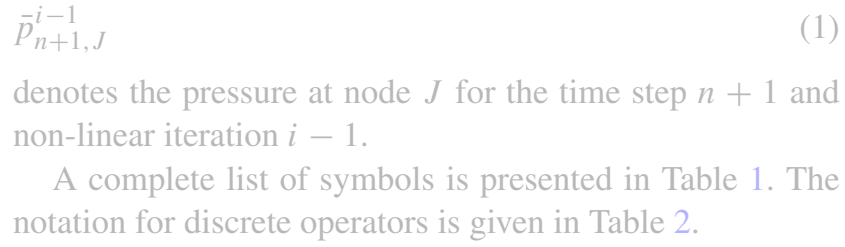

denotes the pressure at node $J$ for the time step $n+1$ and non-linear iteration $i-1$.

A complete list of symbols is presented in Table 1. The notation for discrete operators is given in Table 2 .

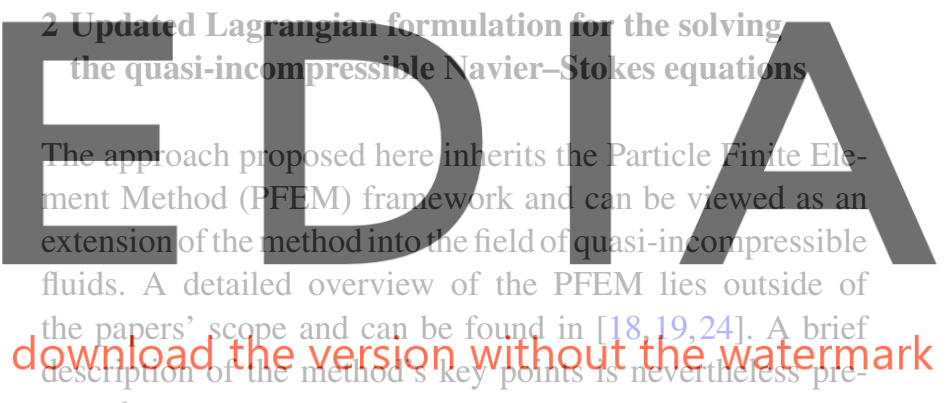

sented next:

\subsection{The particle finite element method concept}

The PFEM adopts a Lagrangian framework for the description of the fluid, where the mesh nodes are treated as particles that can freely move and even separate from the main fluid domain. The fundamental idea of the PFEM is that the variables of interest are stored at the nodes instead of the Gauss points. A finite element mesh is created at every time step of the dynamic problem and the solution is then stored at the nodes. The nodes move according to their velocity obtaining their new position and then a new mesh is created. The generation of the finite element (FE) mesh is done using a Delaunay tessellation [25]. PFEM utilizes linear triangular/tetrahedral meshes. It is important to keep in mind that the convective terms of the momentum equation disappear in the Lagrangian description. Therefore ellipticity and symmetry of the system remains unaltered. Also the stability problems faced in Eulerian methods due to presence of the convective term are not faced here. 
Table 1 List of symbols

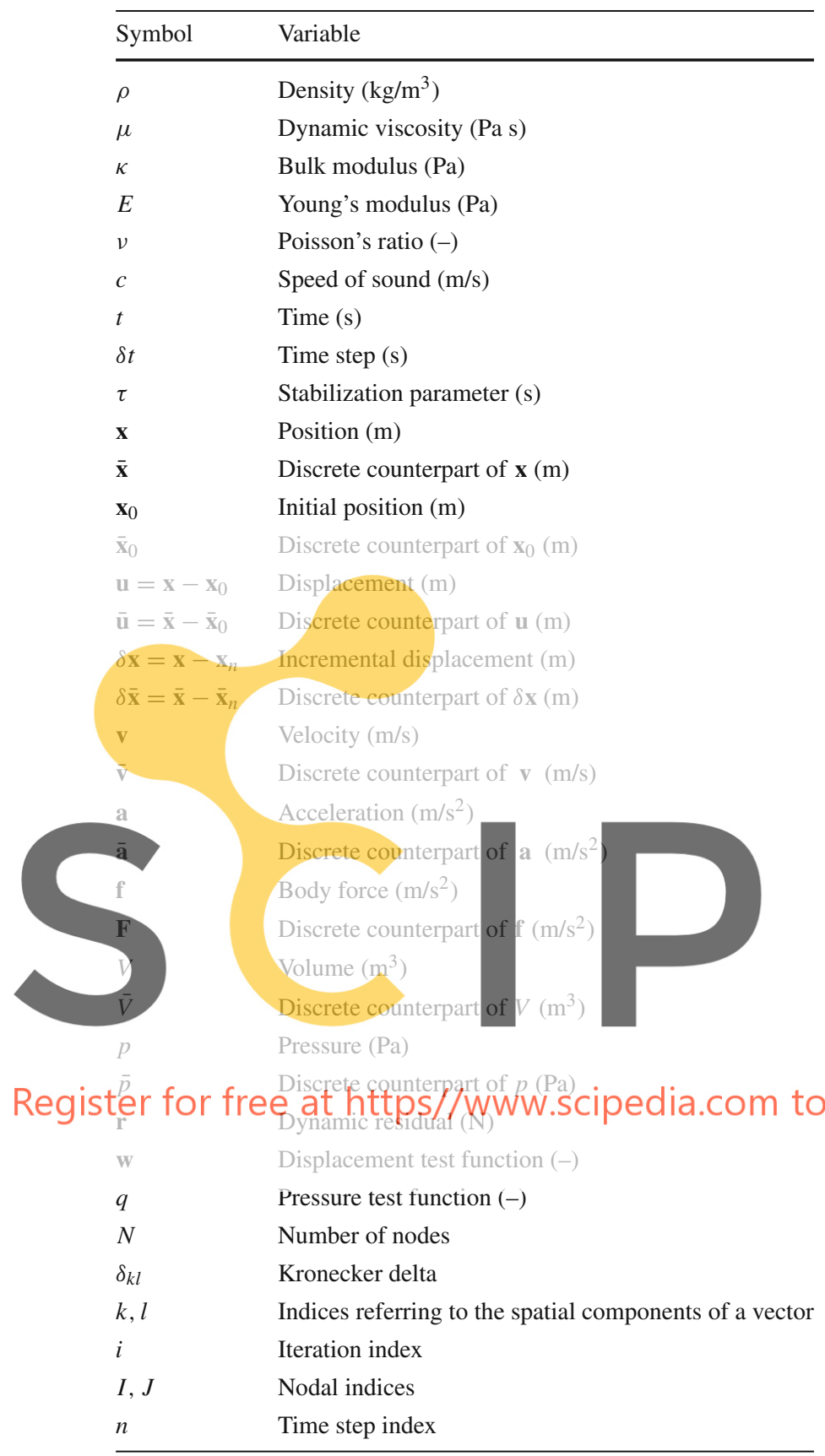

In treating problems involving free surface flows the boundary is determined at every time step using the so-called 'alpha-shape' technique $[18,26]$. This can be seen as a geometric criterion that enables one to decide whether or not a face of an element belongs to the free surface.

\subsection{Assumptions and continuous problem}

Before proceeding further, the terminology utilized shall be clarified. In this work we concentrate on the simulation of "quasi-incompressible" fluids where the term "quasi-
Table 2 Relevant matrices and operators

\begin{tabular}{ll}
\hline Symbol & Discrete operator \\
\hline $\mathbf{B}$ & Strain-displacement matrix \\
$\mathbf{K}$ & Stiffness matrix \\
$\mathbf{M}$ & Lumped displacement mass matrix \\
$\mathbf{M}_{p}$ & Lumped pressure mass matrix \\
$\mathbf{M}_{p}^{c}$ & Consistent pressure mass matrix \\
$\mathbf{D}$ & Divergence operator \\
$\mathbf{G}$ & Gradient operator \\
$\mathbf{L}$ & Laplacian operator \\
$\mathbf{H}$ & Dynamic tangent matrix \\
$\mathbb{C}_{K}$ & Volumetric elasticity tensor \\
\hline
\end{tabular}

incompressibility" implies that thermal influences are negligible and thus the energy equation remains uncoupled from the continuity equation. We accept on the other hand that the fluid is not anymore considered incompressible, meaning that the constraint $(\nabla \cdot \mathbf{v}=0)$ is not satisfied exactly but rather substituted (as we will see later) by a relation between the pressure and the local volume variation.

At this point we introduce the governing equations for a quasi-incompressible Newtonian fluid written in Lagrangian

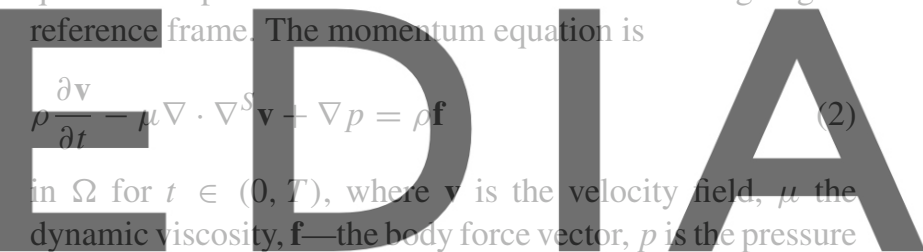
and $\nabla^{S}$ the symmetric gradient operator. Next, we neglect the $\mu /\left(\nabla \cdot v^{N}\right)$ term, assuming that poth the product of fluid down oad the version without the waterm this simplification is only justified for low values of the viscosity. Indeed preserving the exact form does not imply any modification to the theory we will present. In the following we will therefore proceed using the Laplacian form without loss of generality and exclusively for simplifying the notation. Taking into account this observation and introducing the variable $\mathbf{u}=\mathbf{x}-\mathbf{x}_{0}$ we obtain

$\rho \frac{\partial^{2} \mathbf{u}}{\partial t^{2}}-\mu \Delta \frac{\partial \mathbf{u}}{\partial t}+\nabla p=\rho \mathbf{f}$

where $\nabla$ and $\Delta$ stand for gradient and Laplacian operators respectively. The continuity equation in the Lagrangian framework reads

$\frac{\partial \rho}{\partial t}+\rho \nabla \cdot \mathbf{v}=0$

For isothermal flows

$\frac{\partial \rho}{\partial t}=\frac{1}{c^{2}} \frac{\partial p}{\partial t}$

where $c$ is the velocity of sound propagation in the medium. Using Eq. (5) and substituting velocity by the derivative of 
the displacement, we obtain the constitutive equation for the pressure as

$\frac{\partial p}{\partial t}=-\rho c^{2} \nabla \cdot \frac{\partial \mathbf{u}}{\partial t}$

The term $\rho c^{2}$ reflects the fluid compressibility and is called "the bulk modulus", often denoted by $\kappa$. Equation (6) can be written as

$\frac{\partial p}{\partial t}=-\kappa \nabla \cdot \frac{\partial \mathbf{u}}{\partial t}$

An alternative is to relate directly the bulk modulus to the variation of a given control volume $V$.

$\kappa \equiv-\frac{\partial p}{\partial V} V$

The use of such equation is not really appealing at the continuum level, as the definition of "control volume" is somewhat arbitrary. As we will see later however its discrete counterpart will present some important advantages, which will be exploited in the strategy we propose.

The equations that define the fluid motion are Eqs. (2), (6) and (8). At the continuous level Eqs. (7) and (8) are equivalent. Equation (7) relates the temporal derivative of

pressure with the gradient of the velocity field, whereas Ec. (8) expresses the pressure without knowing the temporal curtent configuration. Both equ (8) for pressure recovery once

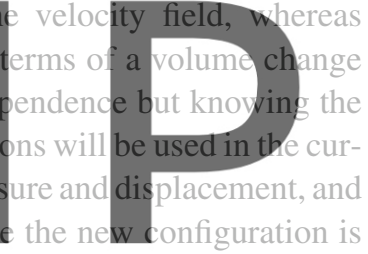

found.

Register for free at https//www.scipedia.com to 2.3 Discretization

The weak form of Eqs. (2) and (7) using a linear displacement-pressure interpolation reads

$$
\begin{aligned}
& \left(\rho \frac{\partial^{2} \mathbf{u}}{\partial t^{2}}, \mathbf{w}\right)+\mu\left(\nabla \frac{\partial \mathbf{u}}{\partial t}, \nabla \mathbf{w}\right)+(\nabla p, \mathbf{w})=\rho\langle\mathbf{f}, \mathbf{w}\rangle \\
& \left(q, \frac{\partial p}{\partial t}\right)=-\kappa\left(q, \nabla \cdot \frac{\partial \mathbf{u}}{\partial t}\right)
\end{aligned}
$$

where $\mathbf{w}$ and $q$ are the displacement and pressure test functions. In the equations above $(\cdot, \mathbf{w})$ and $\langle\cdot, \mathbf{w}\rangle$ are the standard bilinear and linear forms in $\mathbf{w}$ and $(\cdot, q)$ is the bilinear form in $q$. Choosing an updated Lagrangian description means that the current configuration serves as the reference and thus all the spatial integrals are evaluated in $\Omega(t)$. The semi-discrete equations then read

$$
\begin{aligned}
& \rho \mathbf{M} \frac{\partial^{2} \overline{\mathbf{u}}}{\partial t^{2}}-\mu \mathbf{L} \frac{\partial \overline{\mathbf{u}}}{\partial t}+\mathbf{G} \bar{p}=\mathbf{F} \\
& \mathbf{M}_{p} \frac{\partial \bar{p}}{\partial t}=-\kappa \mathbf{D} \frac{\partial \overline{\mathbf{u}}}{\partial t}
\end{aligned}
$$

where $\mathbf{M}$ is the mass matrix, $\mathbf{M}_{p}$-the pressure mass matrix both used in the lumped format (note that if in the mass matrix super-index "c" is omitted, we assume that it is taken in the lumped format), $\mathbf{L}$ the Laplacian operator, $\mathbf{G}$ the gradient operator and $\mathbf{D}$ the divergence operator. These are assembled from elemental contributions defined as

$$
\begin{aligned}
& \mathbf{M}_{I J l k}=\int_{\Omega_{e}} \delta_{k l}\left(N_{I}, N_{J}\right) d \Omega \\
& \mathbf{M}_{p, I J}=\int_{\Omega_{e}}\left(N_{I}, N_{J}\right) d \Omega \\
& \mathbf{L}_{I J}=\int_{\Omega_{e}}\left(\frac{\partial N_{I}}{\partial x_{k}}, \frac{\partial N_{J}}{\partial x_{k}}\right) d \Omega \\
& \mathrm{G}_{I J k}=\int_{\Omega_{e}}\left(\frac{\partial N_{I}}{\partial x_{k}}, N_{J}\right) d \Omega \\
& \mathbb{D}=G^{T} \\
& \mathbb{F}_{I k}=\int_{\Omega_{e}}\left(N_{I}, \mathbb{f}_{k}\right) d \Omega
\end{aligned}
$$

Equations (11) and (12) are still continuous in time. The

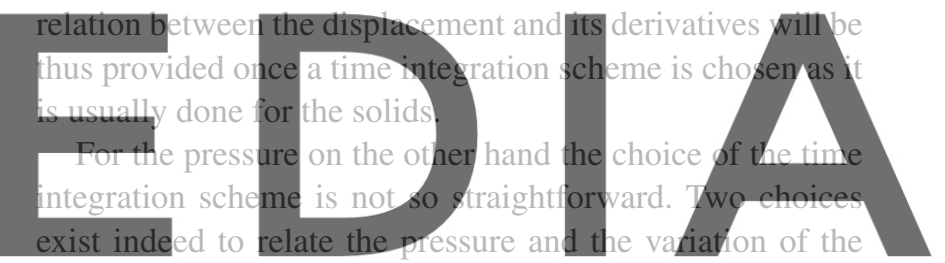
displacement field.

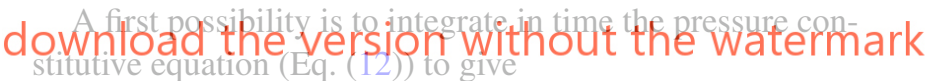

$\mathbf{M}_{p} \delta \bar{p}=-\kappa \int_{t_{n}}^{t_{n+1}} \mathbf{D}(t) \overline{\mathbf{v}} d t$

The difficulty is here that both the velocity and the discrete divergence operator vary in time, making the exact computation difficult to achieve. In the practice some further approximations are typically taken, leading for example to the approximation

$\mathbf{M}_{p} \delta \bar{p}=-\kappa \int_{t_{n}}^{t_{n+1}} \mathbf{D}(t) \overline{\mathbf{v}} d t \approx-\kappa \mathbf{D} \int_{t_{n}}^{t_{n+1}} \overline{\mathbf{v}} d t \approx-\kappa \mathbf{D}_{n+1} \delta \overline{\mathbf{x}}_{n+1}$

This approach is indeed possible, nevertheless it introduces an additional error due to the assumption of the time independence of the divergence operator $\mathbf{D}$, an error that becomes especially considerable for large time steps.

The second option, which we argue to be sensibly better, consists in using Eq. (8), which permits the computation of 
the pressure directly from a given discrete volume change. As commented before Eq. (8) is fully specified only once a definition of the "control volume" is provided. In the following we will associate to each node of the FE mesh a nodal volume defined in such a way that it coincides with the diagonal entry of the pressure lumped mass matrix.

$\bar{V}_{I}:=\mathbf{M}_{p, I I}$

The interesting point is now that, under the hypothesis of constant bulk modulus $\kappa$, the nodal pressure increment can be exactly related to the nodal volume variation by the formula

$\bar{p}_{n+1, J}^{i+1}=\bar{p}_{n, J}-\kappa \frac{\bar{V}_{n+1, J}^{i+1}-\bar{V}_{n, J}}{\bar{V}_{n, J}}$

In this sense Eq. (22) is thus clearly superior to Eq. (20) as it

does not involve any additional approximation.

From the point of view of the practical application on the other hand, we can observe that the nodal volume can be easily computed by nodal integration of the unit function over the volume.

Remark It is interesting to observe that using Eq. (22) can be interpreted as imposing the mass conservation on a num-

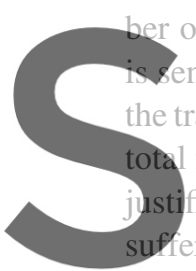
of integration points (the ensibly smaller than the nurn he traditional element based ap otal number of volumetric con ification of the fact that the proposed er locking. This argument

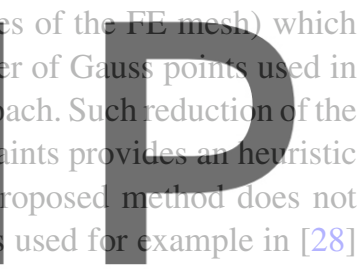

to justify the F-bar method for low order elements.

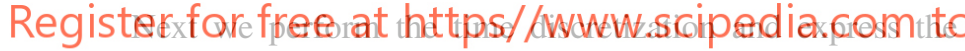
momentum and mass conservation equations in residual form in order to perform next the linearization. For the sake of clarity we shall illustrate the method using the simplest implicit time integration scheme, namely the Backward Euler and only afterwards present the discrete equation in conjunction with Newmark-Bossak scheme. The latter is used for the examples presented at the end of the paper. The momentum residual integrated in time thus gives

$\overline{\mathbf{r}}_{m}:=\mathbf{F}_{n+1}-\mathbf{G} p_{n+1}+\mu \mathbf{L} \frac{\delta \overline{\mathbf{x}}_{n+1}}{\delta t}-\rho \mathbf{M} \frac{\delta \overline{\mathbf{x}}_{n+1}-\delta \overline{\mathbf{x}}_{n}}{\delta t^{2}}$

where $\delta \overline{\mathbf{x}}_{n+1}=\overline{\mathbf{u}}_{n+1}-\overline{\mathbf{u}}_{n}$ the incremental displacement. The pressure equation residual reads

$\overline{\mathbf{r}}_{p}:=\bar{p}_{n, J}-\bar{p}_{n+1, J}^{i+1}-\kappa \frac{\bar{V}_{n+1, J}^{i+1}-\bar{V}_{n, J}}{\bar{V}_{n+1, J}^{i+1}}$

which can be written equivalently

$\overline{\mathbf{r}}_{p}:=\mathbf{M}_{p, J J} \bar{p}_{n, J}-\mathbf{M}_{p, J J} \bar{p}_{n+1, J}^{i+1}-\kappa\left(\bar{V}_{n+1, J}^{i+1}-\bar{V}_{n, J}\right)$
For convenience, we finally introduce the symbol

$\overline{\mathbf{r}}:=\left(\begin{array}{c}\overline{\mathbf{r}}_{m} \\ \overline{\mathbf{r}}_{p}\end{array}\right)$

to denote the overall residual.

\subsection{Linearization}

Equation (26) defines a non-linear system as all the operators are written at the unknown configuration $t_{n+1}$. In order to solve this system Newton method is applied for the linearization. For this the residual $\overline{\mathbf{r}}$ and tangent stiffnesses $\mathbf{H}$ need to be established.

By definition the tangent stiffness is the derivative of the residual with respect to the primary variables

$\mathbb{H}=-\frac{\partial \overline{\mathbf{r}}\left(\overline{\mathbf{x}}^{i}, \bar{p}^{i}\right)}{\partial \overline{\mathbf{x}}, \bar{p}}$

The Newton method can be summarized as follows:

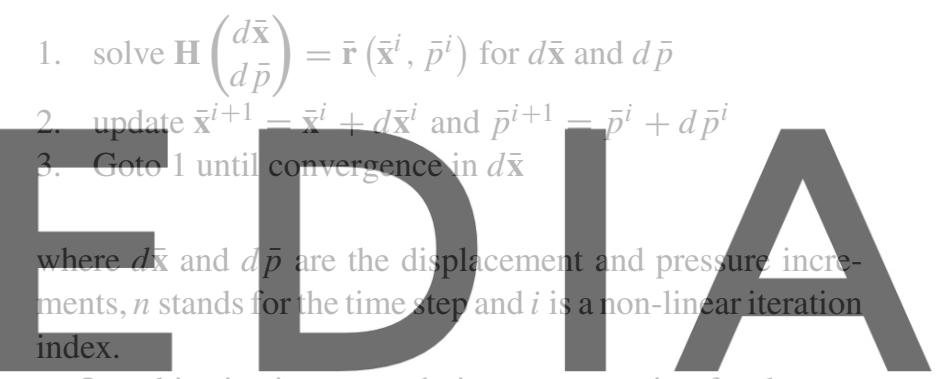

Our objective is now to derive an expression for the tangent stiffness $\mathbb{H}$. The mass matrix and external force vectors d Rwdilqad the versiph dy ithout the waternark

the shape functions and the nodal connectivities do not alter within one time step. The non-linearity is contained in the internal forces, that are the viscous $\mu \mathbf{L} \overline{\mathbf{v}}_{n+1}$ and the volumetric term $\mathbf{G} \bar{p}_{n+1}$. The discrete Laplacian and gradient operators contain derivatives of the shape functions with respect to unknown current configuration $\overline{\mathbf{x}}_{n+1}$. We assume that the discrete operators $\mathbf{L}$ and $\mathbf{G}$ do not change within a non-linear iteration, however this does not imply that they do not change within the time step. At every non-linear iteration $\mathbf{L}$ and $\mathbf{G}$ are recomputed in the newly obtained configuration and then used in the next iteration. So the continuously varying in time $\mathbf{L}(t)$ and $\mathbf{G}(t)$ are approximated within a time step by a series of constant operators $\left(\mathbf{L}^{0}, \mathbf{L}^{1} \ldots \mathbf{L}^{i}\right)$ and $\left(\mathbf{G}^{0}, \mathbf{G}^{1} \ldots \mathbf{G}^{i}\right)$.

The linearization of the pressure residual (Eq. (25)) gives:

$\frac{\partial \overline{\mathbf{r}}_{p}}{\partial p}=\mathbf{M}_{p}$

and

$\frac{\partial \overline{\mathbf{r}}_{p}}{\partial \delta \overline{\mathbf{x}}}=\kappa \frac{\partial\left(\bar{V}_{n+1, J}^{i+1}-\bar{V}_{n, J}\right)}{\partial \delta \overline{\mathbf{x}}}=\kappa \mathbf{D}$ 
which allows to write the Newton system as

$\left(\begin{array}{cc}\rho \frac{\mathbf{M}}{\delta t^{2}}-\mu \frac{\mathbf{L}}{\delta t} & \mathbf{G} \\ \mathbf{D} & \frac{1}{\kappa} \mathbf{M}_{p}\end{array}\right)\left(\begin{array}{c}d \overline{\mathbf{x}}_{n+1} \\ d p\end{array}\right)=\left(\begin{array}{c}\overline{\mathbf{r}}_{m}\left(\overline{\mathbf{x}}^{i}, \bar{p}^{i}\right) \\ \frac{1}{\kappa} \overline{\mathbf{r}}_{p}\left(\overline{\mathbf{x}}^{i}, \bar{p}^{i}\right)\end{array}\right)$

The system described in Eq. (30) is known as a "Monolithic fluid" system (NB: not to be confused with the "monolithic FSI" system.). It couples tightly the pressure and displacements in the sense that the solution for both has to be performed simultaneously. Since a compressible formulation is used a mass matrix (diagonal in our case) appears in the second line of the system.

The following section will be devoted to the discussion of the implications of the proposed method as the incompressible limit is approached. Some problems will be identified in this context, and a modification of the "constitutive" pressure equation will be introduced to palliate such problems.

\subsection{Pressure stability}

The use of mixed approaches cures to a great extent the locking problem and in the compressible regime does not lead to any complications with respect to pressure stability. However as the incompressible regime is approached the insta-

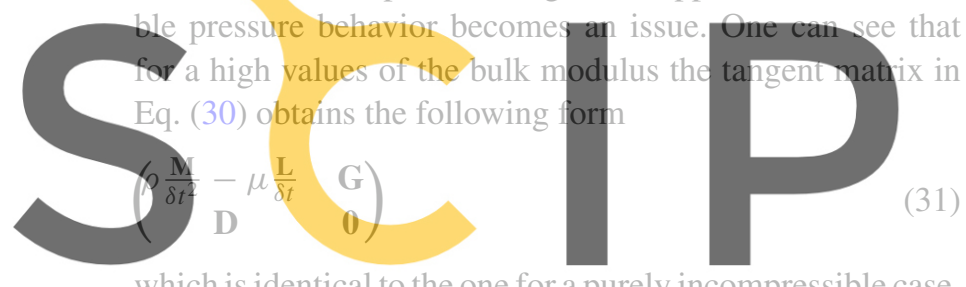

which is identical to the one for a purely incompressible case. For pressure-velpcity pairs that do not pass. the inf-sup Register for free at https / WWWW SGipedia.Gom to more ill-conditioned (eventually indefinite for purely incompressible problems). This in turn implies, by definition of ill-conditioned matrix, that small perturbations in the residuals will lead to arbitrarily large variations in the solution, thus implying that small perturbations in the residuals may eventually grow and destroy the pressure solution.

In order to introduce and provide an heuristic justification of our proposed modification, let us consider a static equilibrium condition under the assumption of zero body force $\mathbf{F}=\mathbf{0}$, and let's assume further that the nodes are distributed in a Cartesian way.

Static solution implies that the variation of displacement from one time step to the following is zero. On the other hand at equilibrium $p_{n} \equiv p_{n+1}^{i+1}$ and $\bar{V}_{n+1}^{i+1} \equiv \bar{V}_{n}$. We can now make the following observations:

- Since the operator $\mathbf{M}_{p}$ acts on both $p_{n+1}$ and $p_{n}$, independently of the spatial distribution of pressure, a pressure that does not vary in time will guarantee that the pressure residual will be identically equal to zero (of course in the hypothesis that the volume does not change).
- For the Cartesian distribution assumed, different spatial pressure distributions exist that make zero the residual of the momentum equation, for example a constant (zero) pressure and a spurious check-board like mode (see e.g. [30] or [31]).

These last two observations are enough to prove that multiple solutions may exist, unless $\mathbf{M}_{p}$ is modified so to introduce some spatial relation between the pressure on neighboring nodes. For incompressible fluids the pressure is commonly stabilized by the introduction of a "pressure Laplacian". Even if this is optimal in the context of monolithic techniques, we do not follow this approach in current work, since we like the pressure term to be diagonal.

Our proposal to palliate the problem is to modify Eq. (25) as

$\overline{\mathbf{r}}_{p}=\mathbf{M}_{p}^{c} \bar{p}_{n}-\mathbf{M}_{p} \bar{p}_{n+1}^{i}-\kappa\left(\bar{V}_{n+1}^{i+1}-\bar{V}_{n}\right)$

where $\bar{p}_{n}$ is weighted by a consistent pressure mass matrix rather that by the lumped version. The key point here is that, at steady state Eq. (32) simplifies to

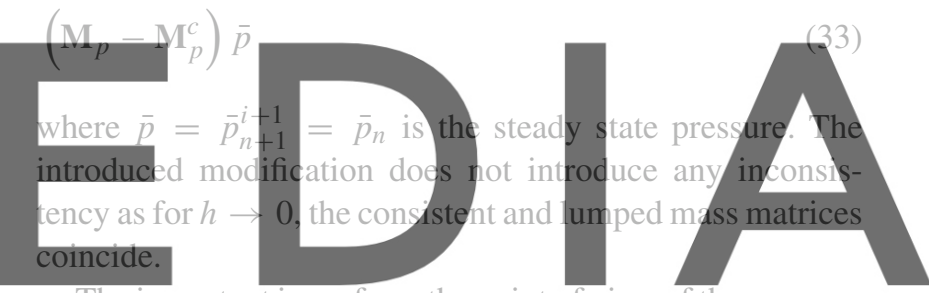

The important issue from the point of view of the pressure stability is that the modified form introduces an additional dawnlpad the versian without the watermark

is now called to guarantee that $\left(\mathbf{M}_{p}-\mathbf{M}_{p}^{c}\right) \bar{p}=0$.

Since the operator $\mathbf{M}_{p}^{c}-\mathbf{M}_{p}$ approximate the Laplacian [32] (in 1D it can be shown to be exactly equivalent), the proposed stabilization can be thus viewed (at steady state) as a simple Laplacian-type stabilization, ensuring some degree of pressure stability.

Remark The stabilization effect achieved is still weak for very high values of the bulk modulus. This can be easily seen by considering that the "stabilization term" is weighted by $\frac{1}{\kappa}$ which implies that $\kappa \rightarrow \infty$ the instable equation is recovered. We argue nevertheless that the modification proposed in very effective in the practice, for a large range of the bulk modulus values.

\subsection{Global pressure condensation}

Up to this moment we have to defined a monolithic system of equations which "tightly" relates the displacements of the nodes with the pressure. By "tight" relation we mean that the system is fully coupled, namely none of the entries of the 
tangent stiffness is zero, thus requiring simultaneous solution for the velocity and pressure. Such system can be easily constructed and allows in principle the use of any stabilization technique. However the monolithic system defined by Eq. (30) does not provide any significant advantage in comparison with the standard incompressible one. The poor conditioning even for the single field problems would require utilization of direct solvers.

In order to deal with large problems we take advantage of the introduced compressibility and symbolically condense the pressure from the global system in order to obtain a displacement-only system that is equivalent to the original linear system. As we shall see later, this is possible if pressure is stabilized using the method described above, while it would be impossible if a Laplacian type stabilization was used.

The symbolic condensation of the system in Eq. (30) gives

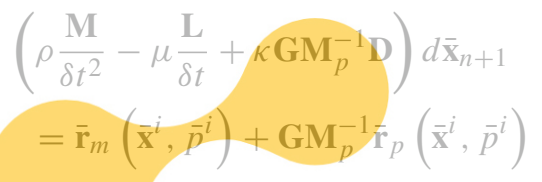

(34)

This is practically implemented as the equivalent multi-step procedure:

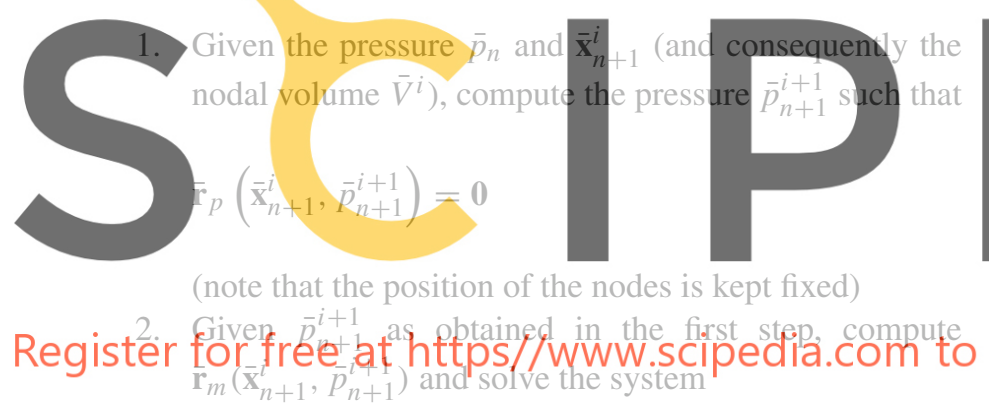

$$
\left(\rho \frac{\mathbf{M}}{\delta t^{2}}-\mu \frac{\mathbf{L}}{\delta t}+\kappa \mathbf{G M}_{p}^{-1} \mathbf{D}\right) d \overline{\mathbf{x}}_{n+1}=\overline{\mathbf{r}}_{m}\left(\overline{\mathbf{x}}^{i}, \bar{p}_{n+1}^{i}\right)
$$

3. Update the nodal position as

$$
\overline{\mathbf{x}}_{n+1}^{i+1}=\overline{\mathbf{x}}_{n+1}^{i}+d \overline{\mathbf{x}}_{n+1}
$$

4. If not converged go back to step 1

The only system of equation to be solved is thus found in the second step. Introducing the notation $\mathbf{H}_{f}=\rho \frac{\mathbf{M}}{\delta t^{2}}-$ $\mu \frac{\mathbf{L}}{\delta t}+\mathbf{G M}_{p}^{-1} \mathbf{D}$ to denote the tangent stiffness of the fluid corresponding to the momentum equation we can write

$\mathbf{H}_{f} d \overline{\mathbf{x}}=\overline{\mathbf{r}}_{m}$

which is written exclusively in terms of displacements.

Remark Note that pressure condensation did not require solution of any system of equations due to the lumped format of the pressure mass matrix In the case a classical pressure stabilization matrix was added to the monolithic system, such global condensation would have been impossible due to the enormous effort of symbolically inverting a non-diagonal sparse matrix.

\subsection{Coupling with the structure}

In the following "monolithic FSI" means a discrete equation system that includes the equations of both the sub-domains. It should not be confused with the term "monolithic singlefield" that was used when describing the fluid formulation, where it referred to simultaneous solution for the primary variables (velocity/pressure).

Here we shall present the coupling strategy with the structure and establish the monolithic FSI system. The illustration will be performed without specifying any particular type of structure. It can be a solid, membrane, rotation-free shell or any other FE structure. The only prerequisite is that the discrete equations are written in terms of displacements. We note, that the time integration scheme must be consistent with the one used for the fluid. The discrete momentum equations for the solid in the absence of damping, using backward Euler time integration can be written as

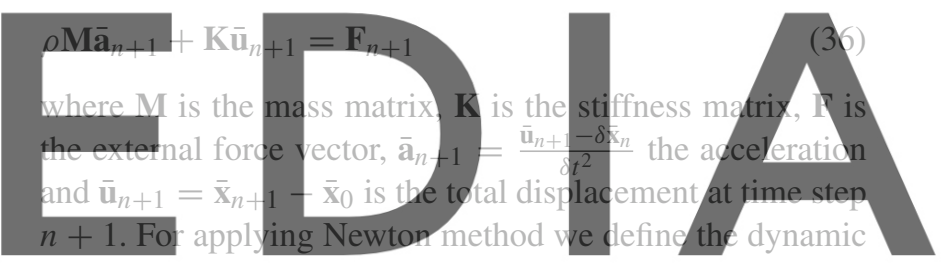

residual and tangent stiffness

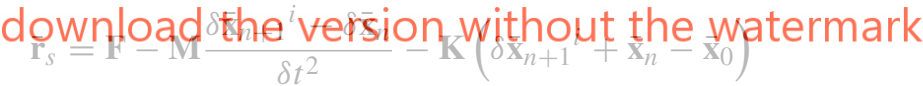

$\mathbf{H}_{s}=\frac{\partial \overline{\mathbf{r}}_{s}}{\partial \delta \overline{\mathbf{x}}}$

where subscript $s$ stands for "structure". The tangent matrix and the residual for the fluid given by Eqs. (35) and (23) will be distinguished by subscript $f$. We shall split the dynamic tangent stiffness of the fluid into two parts for reasons that will become clear later.

$\mathbf{H}_{f}=\mathbf{H}_{f}^{a}+\mathbf{H}_{f}^{b}$

where

$\mathbf{H}_{f}^{a}=\mathbf{M}-\mu \mathbf{L}$

and

$\mathbf{H}_{f}^{b}=-\kappa \mathbf{G M}_{p}^{-1} \mathbf{D}$

The linearized monolithic FSI equations system is obtained then in two steps: first a standard FE assembly procedure is carried out by looping over all the elements (fluid and structure). Structure elements contribute $\overline{\mathbf{r}}_{s}$ and $\mathbf{H}_{s}$ 
whereas fluid elements contribute $\overline{\mathbf{r}}_{f}$ and $\mathbf{H}_{f}^{a}$ to the unique FSI dynamic residual and tangent stiffness $\overline{\mathbf{r}}_{F S I}$ and $\mathbf{H}_{F S I}$, respectively.

Then $\mathbf{G M}_{p}^{-1} \mathbf{D}$ needs to be added. The monolithic FSI linearized system can then be written as

$$
\left(\mathbf{H}_{F S I}-\kappa \mathbf{G M}_{p}^{-1} \mathbf{D}\right) d \overline{\mathbf{x}}=\overline{\mathbf{r}}_{F S I}
$$

Note that the $\mathbf{G M}_{p}^{-1} \mathbf{D}$ is a global matrix defined for the fluid only. Its entries corresponding to the degrees of freedom of the structure nodes are zero.

\subsection{Computational efficiency}

The addition of the $\mathbf{G M}_{p}^{-1} \mathbf{D}$ term is a computationally expensive procedure. The costs associated with construction and storage of this matrix at each Newton-Raphson would make the method unfeasible for practical applications. The is easy understandable as the resulting matrix is densely populated and its computation involves sparse matrix-matrix products. We propose to treat the problem defined by Eq. (42) by iterative technique. The choice of using Krylov-type methods implies that a matrix-free approach can be used, thus

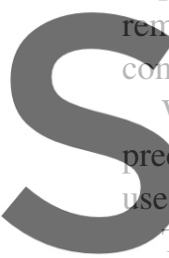

removing the need of construct

computationally expensive GI

We shall illustrate the metho

conditioned Conjugate Grac

in our applications).

The main idea of matrix-free approaches is that only the

product $\mathrm{GM}_{p}^{-1} \mathbf{D} d \overline{\mathbf{x}}$ needs to be known, that is, we need to

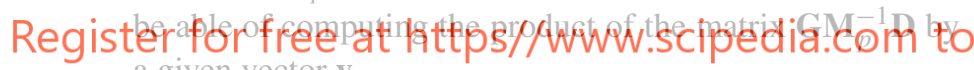
a given vector $\mathbf{y}$.

This can be easily obtained by a sequence of matrix-vector products in the form
1. $\mathbf{e}_{1}=\mathbf{D} d \overline{\mathbf{x}}$
2. $\mathbf{e}_{2}=\mathbf{G e}_{1}=\mathbf{D}^{T} \mathbf{e}_{1}$
3. $\mathbf{e}_{3}=\mathbf{M}_{p}^{-1} \mathbf{e}_{2}$

Taking advantage that $\mathbf{G}=\mathbf{D}^{T}$ we thus need only to construct and store the discrete divergence operator $\mathbf{D}$. Ultimately instead of computing three matrix-matrix products we end up computing two matrix vector products (assuming $\mathbf{M}_{p}$ being a lumped matrix). Apart from the memory efficiency of this approach, it also turns out that, due to the structure of the $\mathbf{D}$ operator, the application of this technique requires less operations than the application of the assembled matrix. The speed up obtained by doing this in comparison with direct computation of $\mathbf{G M}_{p}^{-1} \mathbf{D}$ results to be of $\sim 3$ in $2 \mathrm{D}$ and $\sim 7$ in $3 \mathrm{D}$. This speed up refers to the solution of linearized system performed at each non-linear iteration. Details of the speed up calculation are given in Appendix 1.
Table 3 Implementation procedure for the solution of the monolithic FSI involving quasi-incompressible updated Lagrangian fluid

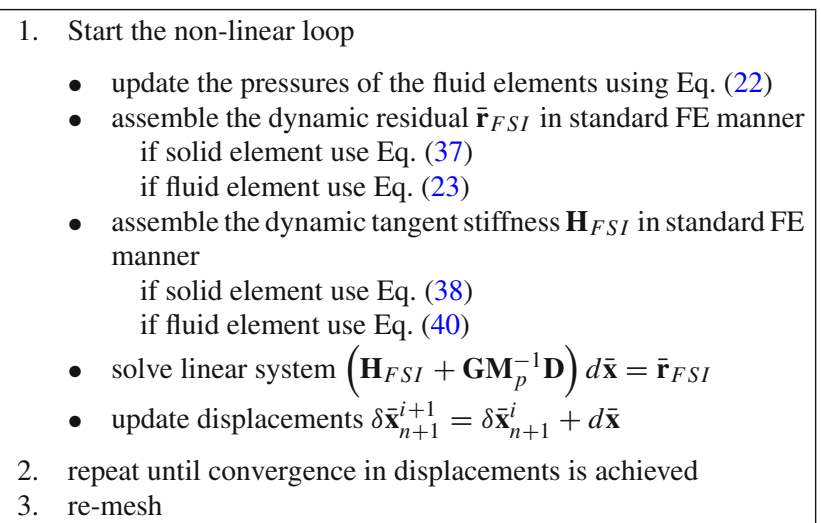

In Eq. (30) the displacements of the interface nodes (i.e. the ones that belong both to the fluid and the solid) are automatically multiplied by the sum of the respective contributions of the fluid and solid dynamic tangent stiffnesses. The system is then treated exactly in the same way as a single field one, i.e. the solver does not "see" the difference between the fluid and the solid and no interface equation needs to be

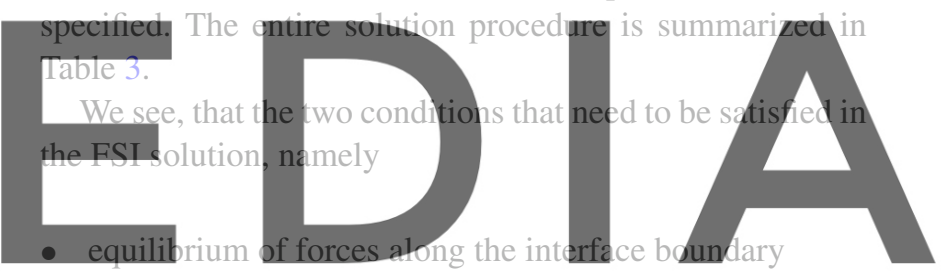

- continuity of displacements along the interface boundary

\section{download the version without the watermark} are satisfied automatically. The computational cost introduced by iterations for satisfaction of these two conditions in a partitioned approach is shifted to the quality of convergence in a monolithic approach.

Remark The computational cost associated with the solution of each time step will depend on two factors:

- Number of non-linear iterations

- Time spent per one non-linear iteration or, equivalently number of the linear solver iterations

These two criteria will be considered in the assessment of the method presented in the next section of the paper.

\section{Examples}

This section shows functionality of the formulation. The formulation was implemented in the Kratos Multi-Physics System, a C++ object oriented FE framework [33]. 
Fig. 1 Dam break problems: a fluid only, b FSI (a)

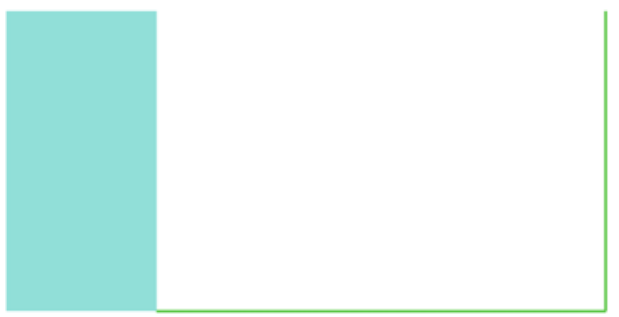

(b)

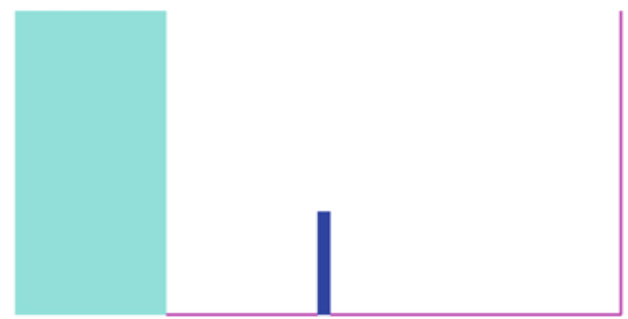

\subsection{Dam break example}

\subsubsection{Practical applicability}

The quality of a monolithic method is strongly related to the convergence characteristics it provides. The proposed formulation does not suffer from volumetric locking in comparison with the quasi-incompressible formulations or penalty methods that use discontinuous pressure. Therefore the bounds that can provide us the practically admissible values of bulk modulus are related to the convergence speed that will be discussed here. Two types of convergence are considered. First is the convergence of Newton method, which reflects the quality of the propc

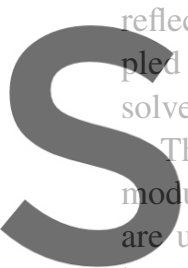

system. Second issue is th olver.

he latter is expected to be affected by

dulus to a great extent. Gener used, the conditioning of
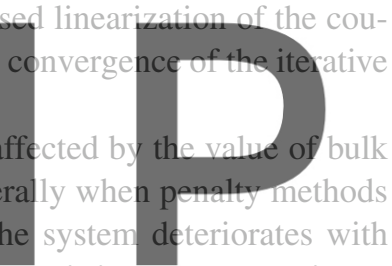

increasing of the penalty constant. It is important to estimate how much the value of bulk modulus affects the convergence

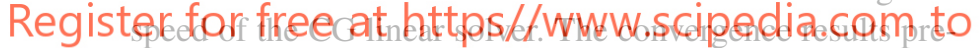
sented next do not pretend to be exhaustive or to provide a clear limit for the value of bulk modulus one can use. Rather they show the tendency and give a qualitative outlook of the convergence behavior. We shall get an insight of the convergence speed by trying both strict and mild convergence requirements.

The example chosen deals with the callapse of a water column. The case including an elastic obstacle as proposed in [34] as well as the case without an obstacle are analyzed. The model geometries are depicted in Fig. 1. The properties of the elastic obstacle are as follows:

- width $w=0.012 \mathrm{~m}$

- height $h=0.08 \mathrm{~m}$

- density $\rho=2,500 \mathrm{~kg} / \mathrm{m}^{3}$

- Young's modulus $E=10^{6} \mathrm{~Pa}$

- Poisson's ratio $v=0$

Dimensions of the water column are: $w=0.146 \mathrm{~m}$ and height $H=0.292 \mathrm{~m}$. The distance between the walls is $b=0.584 \mathrm{~m}$.
Table 4 Convergence characteristics: $\delta t=0.001 \mathrm{~s}, 1,000$ elements

\begin{tabular}{lrlclc}
\hline$\kappa$ & \multicolumn{1}{c}{$c$} & N-R (fluid) & CG (fluid) & N-R (FSI) & CG (FSI) \\
\hline 10E05 & 10 & 3.96 & 25 & 4.12 & 63 \\
$10 \mathrm{E} 06$ & 33 & 4.24 & 68 & 4.2 & 115 \\
$10 \mathrm{E} 07$ & 100 & 4.59 & 200 & 4.89 & 323 \\
$10 \mathrm{E} 08$ & 333 & 4.91 & 460 & 4.93 & 816 \\
\hline
\end{tabular}

Table 5 Convergence characteristics: $\delta t=0.001 \mathrm{~s}, 30,000$ elements

\begin{tabular}{llllll}
\hline$\kappa(\mathrm{Pa})$ & $c(\mathrm{~m} / \mathrm{s})$ & $\mathrm{N}-\mathrm{R}$ (fluid) & $\mathrm{CG}$ (fluid) & $\mathrm{N}-\mathrm{R}(\mathrm{FSI})$ & $\mathrm{CG}(\mathrm{FSI})$ \\
\hline
\end{tabular}

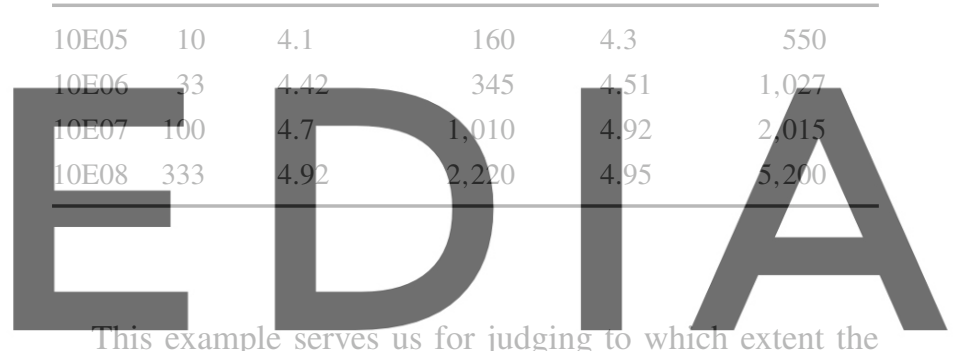

coupling with the structure slows down the computation in dowpload the versiomiwnithouththeouvatermark

values of bulk modulus were used. Two meshes were considered, one containing $\approx 1,000$ linear triangles and the other $\approx 30,000$ linear triangles. First, we examine the convergence using a strict criterion: $\frac{\|d \overline{\mathbf{x}}\|}{\left\|\delta \overline{\mathbf{x}}_{n+1}\right\|}<10 \mathrm{E}-06$ for the non-linear loop and $\frac{\left\|\overline{\mathbf{r}}-\mathbf{H} d \delta \overline{\mathbf{x}}_{\Phi}\right\|}{\left\|\overline{\mathbf{r}}-\mathbf{H} d \delta \overline{\mathbf{x}}_{0}\right\|}<10 \mathrm{E}-08$ for the Conjugate Gradient linear solver. Iterations number (averaged over the simulation time span 0-1 s) for both the non-linear loop and linear solver are given for both purely fluid problem and FSI problem in the Table 4 (rough mesh) and Table 5 (fine mesh). The notation is as follows: $c$ is the sound speed corresponding to the bulk modulus $\kappa$, "N-R" and "CG" are the number of nonlinear (Newton-Raphson) and linear solver (CG) iterations respectively. "Fluid" refers to the purely fluid problem, "FSI" refers to water column against elastic obstacle problem.

These results prove that the convergence of the non-linear (Newton-Raphson) loop does not deteriorate much as the value of the bulk modulus increases. Basically two "ingredients" of the dynamic tangent are competing - the inertia term (inversely proportional to the time squared) and the volumetric term. We assume that the influence of the viscous term upon the non-linear convergence behavior is negligible for 
Table 6 Convergence characteristics for a linear solver tolerance of $10^{-4}, \delta t=0.001 \mathrm{~s}, 1,000$ elements

\begin{tabular}{cclclc}
\hline$\kappa(\mathrm{Pa})$ & $c(\mathrm{~m} / \mathrm{s})$ & $\mathrm{N}-\mathrm{R}$ (fluid) & $\mathrm{CG}$ (fluid) & $\mathrm{N}-\mathrm{R}$ (FSI) & $\mathrm{CG}(\mathrm{FSI})$ \\
\hline $10 \mathrm{E} 05$ & 10 & 2 & 7 & 2 & 32 \\
$10 \mathrm{E} 06$ & 33 & 2 & 18 & 2 & 73 \\
$10 \mathrm{E} 07$ & 100 & 2 & 40 & 2 & 250 \\
$10 \mathrm{E} 08$ & 333 & 2 & 102 & 2 & 626 \\
\hline
\end{tabular}

Table 7 Convergence characteristics for a linear solver tolerance of $10^{-4}, \delta t=0.001 \mathrm{~s}, 30,000$ elements

\begin{tabular}{cclclc}
\hline$\kappa(\mathrm{Pa})$ & $c(\mathrm{~m} / \mathrm{s})$ & $\mathrm{N}-\mathrm{R}$ (fluid) & $\mathrm{CG}$ (fluid) & $\mathrm{N}-\mathrm{R}(\mathrm{FSI})$ & $\mathrm{CG}(\mathrm{FSI})$ \\
\hline $10 \mathrm{E} 05$ & 10 & 2 & 22 & 2 & 180 \\
$10 \mathrm{E} 06$ & 33 & 2 & 50 & 2 & 435 \\
$10 \mathrm{E} 07$ & 100 & 2 & 180 & 2 & 642 \\
$10 \mathrm{E} 08$ & 333 & 2 & 250 & 2 & 1,250 \\
\hline
\end{tabular}

low values of viscosity such as that of water. Small time steps lead to domination of the inertia term $\frac{1}{\delta t^{2}} \mathbf{M}$ in the tangent stiffness, whereas high values of bulk modulus increase the impact of the volumetric term $\kappa \mathbf{G M}_{p}^{-1} \mathbf{D}$. As mentioned in Sect. 2.4, the inertia term is linearized exactly as the mass matrix does not change within one time step.

Note that the linearization that lead to the definition of the monolithic system in Eq. (30) is not entirely exact as the exact linearization would imply (for example) the derivation of the $\mathbf{G}$ operator with respect to the displacement of the nodes. Nevertheless the optimal convergence rates of Newton-Raphson method are assured if the time step is sufficiently small.

Results reported in Tables 2, 4 and 5, confirm that the optimal convergence speed for the non-linear problem is retained both for the case of fluid only and considering complete FSI problems. This indicates that the linearization of the monolithic system is done correctly, and in particular the presence of the structure and thus the interaction does not deteriorate the convergence of the non-linear procedure. For both coarse and fine discretization convergence is achieved in average within $\sim 3-4$ non-linear iterations.

Results also show how the number of iterations of the linear solver grows as $\kappa$ increases, which is associated with the impoverishing conditioning of the linear system to be solved. The results provided here indicate that a preconditioner better than the one utilized here (diagonal preconditioner) could improve the convergence rates of the linear solver.
Fig. 2 Dam break against an elastic obstacle: a $0.0 \mathrm{~s}, \mathbf{b} 0.15 \mathrm{~s}$, c $0.25 \mathrm{~s}, \mathbf{d} 0.45 \mathrm{~s}$, e $1.0 \mathrm{~s}, \mathbf{f} 2.5 \mathrm{~s}$ (a)

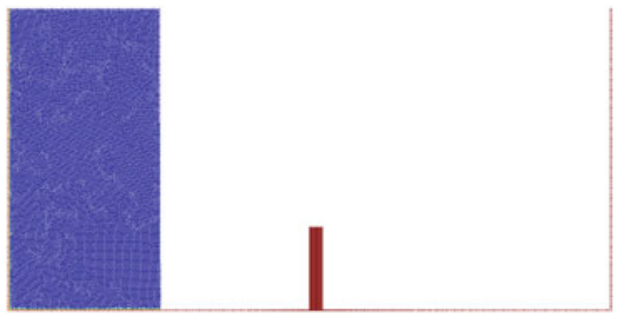

(c)

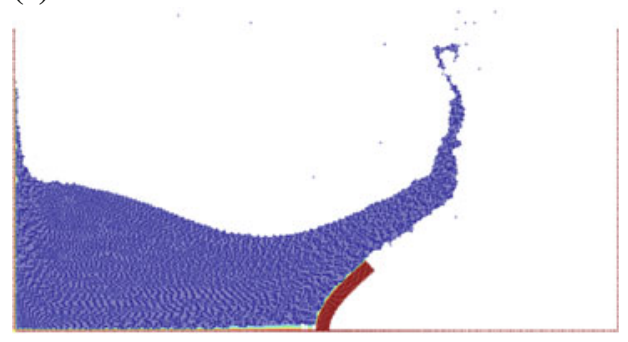

(e)

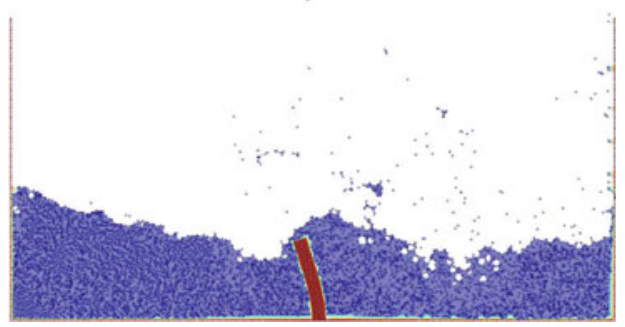

(b)

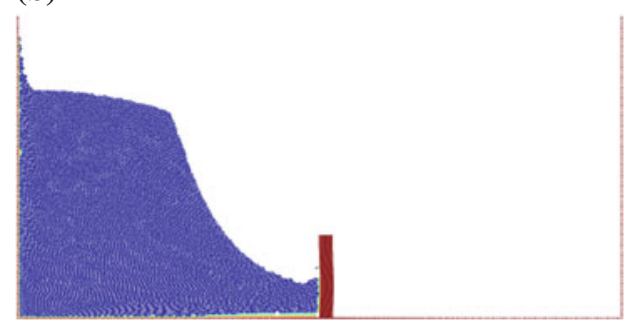

(d)

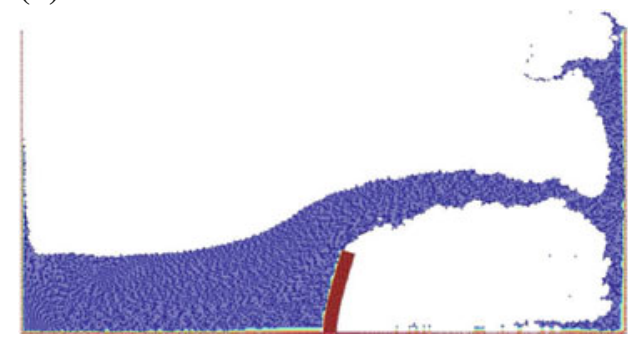

(f)

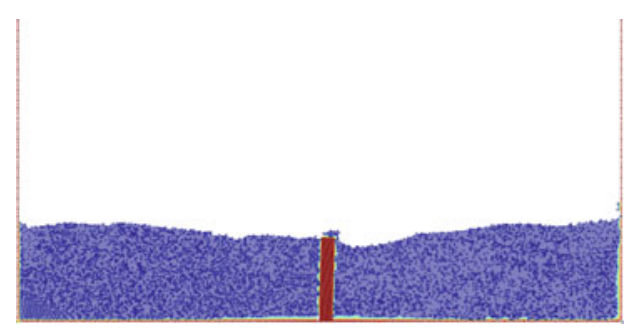




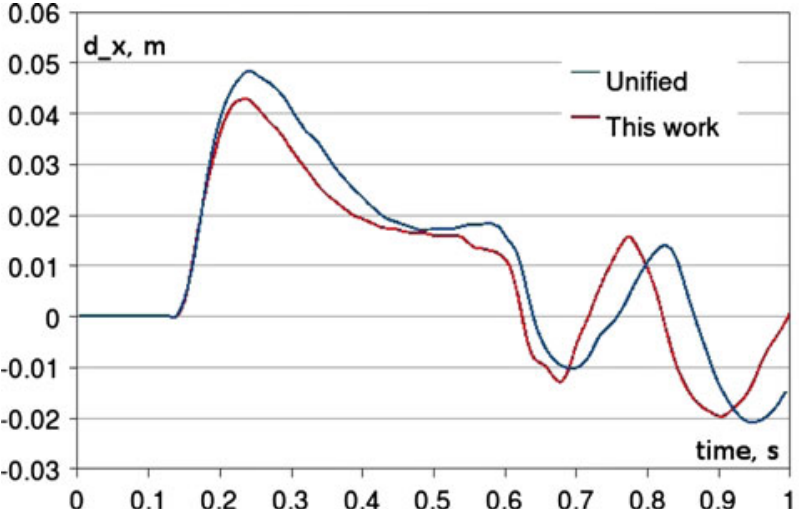

Fig. 3 Dam break against an elastic obstacle: $d_{x}$ comparison of the current approach with the results of [21]

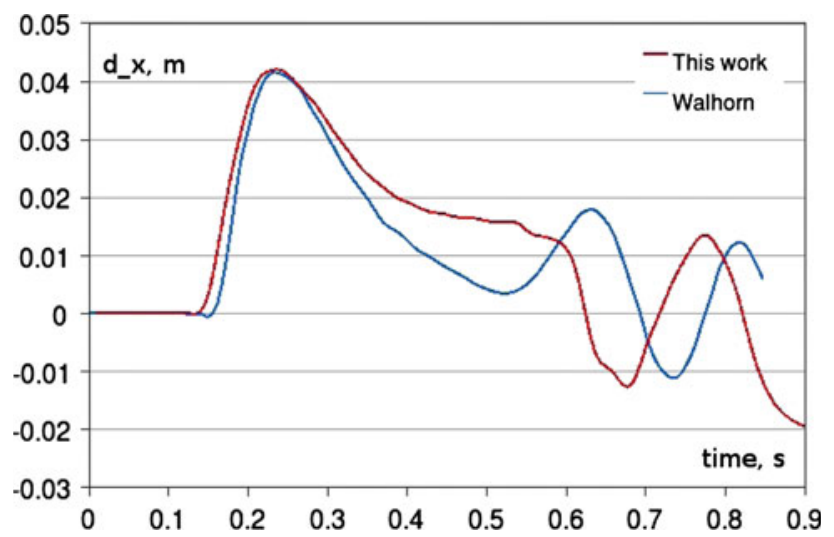

Fig. 4 Dam break against an elastic obstacle: $d_{x}$ comparison of the proposed approach with the results of [35]

It is also important to observe that the number of iterations needed for the convergence of the CG differs in FSI problem (where elastic obstacle is included) by a factor of $\sim 2-2.5$ from that of the fluid-only problem on both the meshes. This is justified by a difference in stiffness between the structural part and the fluid domain.

For practical FSI applications, the strict convergence requirements chosen in the above-mentioned tests are unnecessary. In Tables 6 and 7 we present the convergence data under the following criterion: $10^{-4}$ for both the non-linear and the iterative solvers. Again, two meshes were considered: the coarse one (1,000 elements) and the fine one (30,000 elements). One can see, that the non-linear solver requires two iterations for any examined value of the bulk modulus. It is important to note that under the milder convergence criterion the non-linear solver convergence did not deteriorate.

Remark As mentioned above it is possible to set-up and solve directly a monolithic fluid displacement-pressure system according to Eq. (30). The implementation of this formulation has shown that the Conjugate Gradient solver with diagonal preconditioner fails to resolve the problem already for the bulk modulus value of $\approx 100,000 \mathrm{~Pa}$ with the mesh

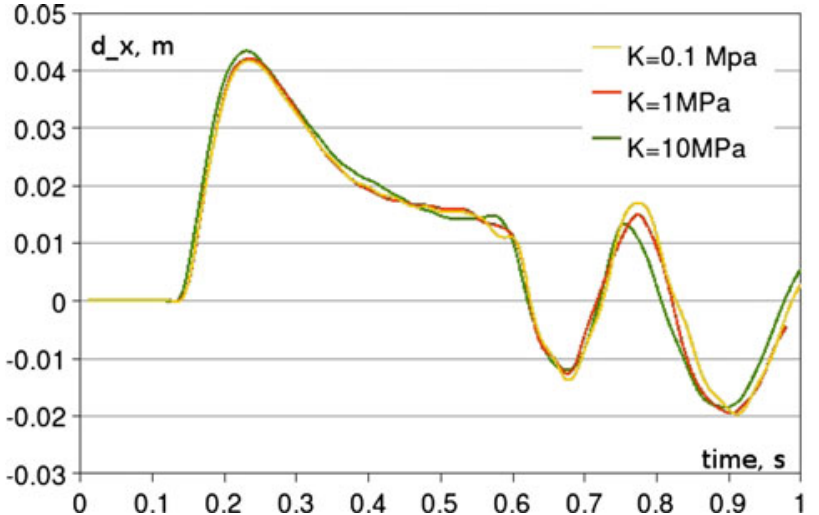

Fig. 5 Dam break against an elastic obstacle: $d_{x}$ comparison of the results obtained for different values of bulk modulus

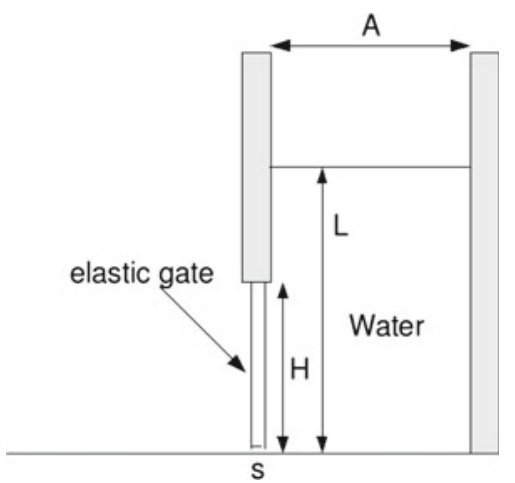

Fig. 6 Deformation of an elastic plate subjected to water pressure

and time step size equivalent to the one used above. Only the application of a direct solver (Super LU in this case) circumvents the problem for practically any value of the bulk modulus. Unfortunately this cannot be applied for the real-life problems involving hundreds of thousands or more degrees of freedom. This fact shows the advantage achieved when pressure condensation is applied. The implementation of the monolithic system also allowed verifying that the result obtained by the monolithic approach are identical to the ones achieved by our pressure condensation algorithm.

\subsubsection{Validation and comparison with other methods}

Here we shall compare the results obtained using the present formulation with the published results obtained by different methods. The comparison is made considering the temporal evolution of the horizontal displacement $d_{x}$ of the upper left corner of the elastic obstacle.

Figure 2 presents snapshots of the simulation using the proposed method, taken at various time instances.

Solutions of this problem obtained in [21] and the one obtained with the approach proposed here are presented in Fig. 3. Bulk modulus for modeling quasi-incompressibility 
Fig. 7 Deformation of an elastic plate subjected to water pressure: comparison with experiment
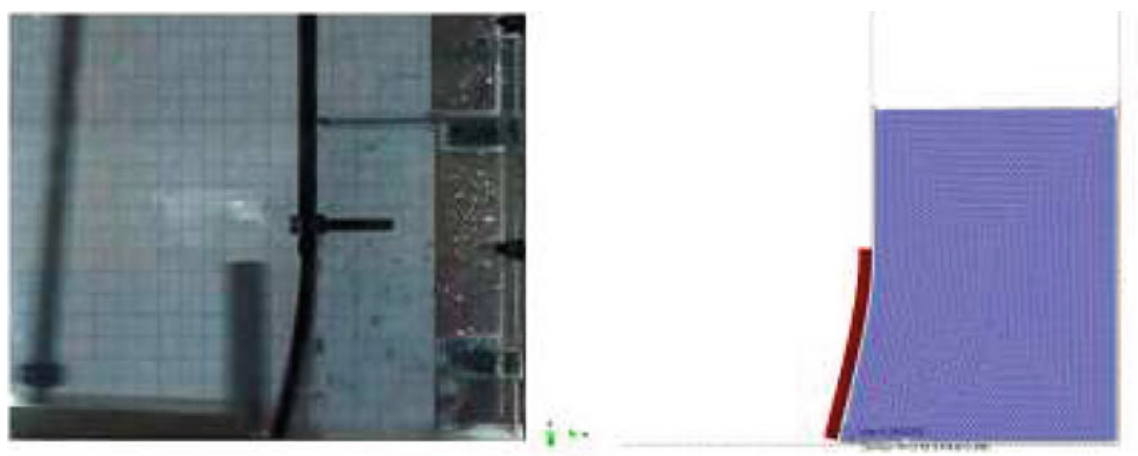

$0.04 \mathrm{~s}$
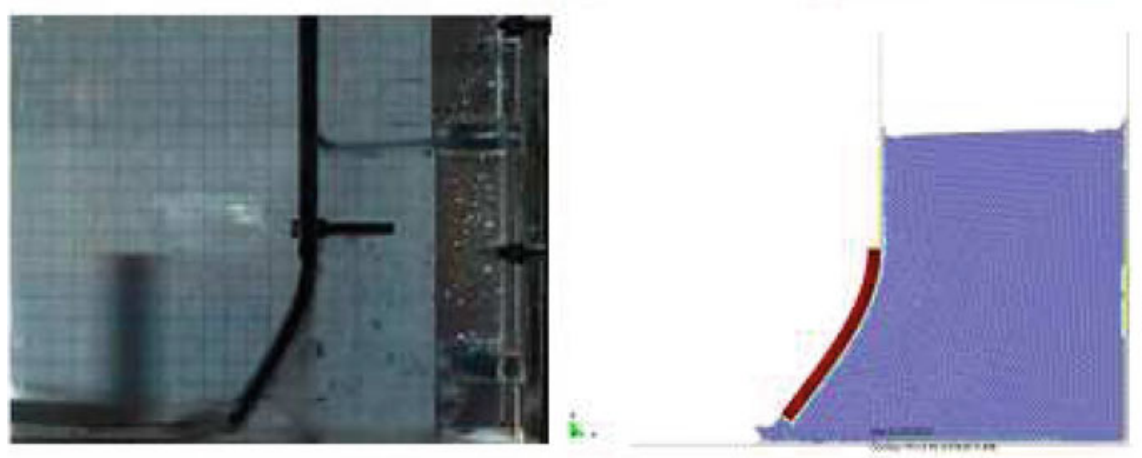

$0.08 \mathrm{~s}$
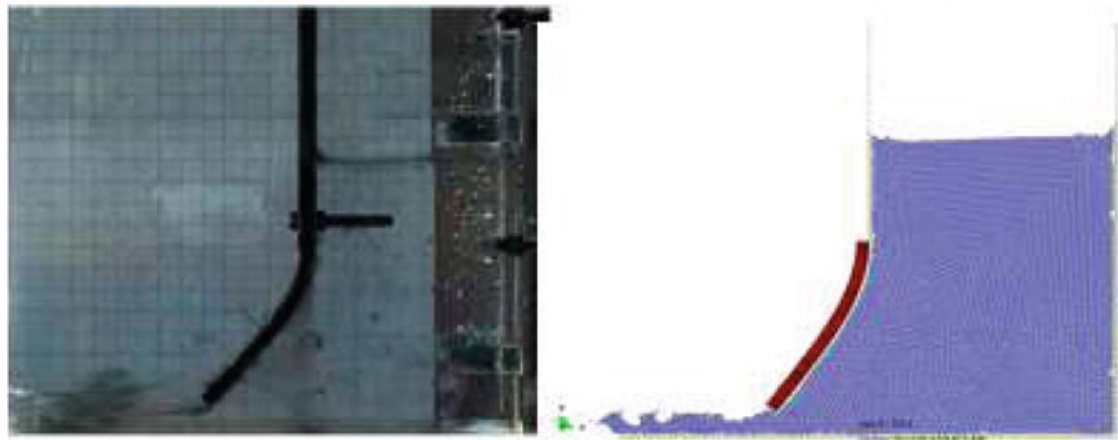

$0.12 \mathrm{~s}$

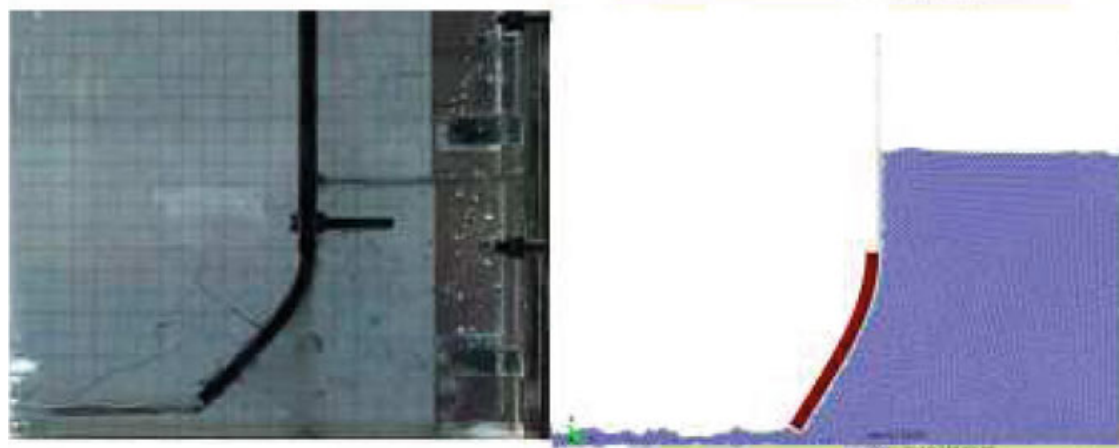

$0.16 \mathrm{~s}$ behavior was set to $\kappa=10^{5}$ Pa for unified formulation [21] and $\kappa=10^{7} \mathrm{~Pa}$ in case of the formulation proposed here. Figure 3 depicts the temporal evolution of the horizontal displacement component of the upper right corner of the obstacle. The total time considered in the simulation was $2.5 \mathrm{~s}$. Good agreement is obtained with results of [21].

Another comparison is made with the space-time monolithic approach with level-set method for the free surface identification published in [35]. There water is modeled as incompressible and the obstacle as a hyper-elastic material. The comparison is depicted in Fig. 4.

Finally, the effect of the bulk modulus upon the solution of the problem has been studied. In Fig. 5 the displacement evolution of the upper right corner of the elastic obstacle obtained using different values of the bulk modulus is shown. One can see good agreement between the graphs. In particular, this ensures that the method is fairly insensitive to the value of the bulk modulus in the sense of repre- 
Fig. 8 Deformation of a membrane filled with water: a initial shape, $\mathbf{b}$ maximum deformation, c equilibrium state, d $3 \mathrm{D}$ view of max deformation

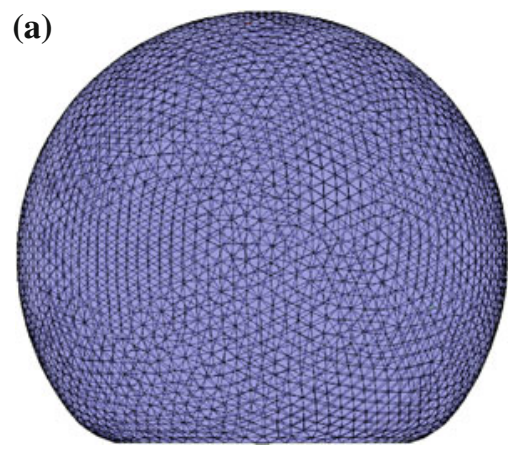

(b)

(c)

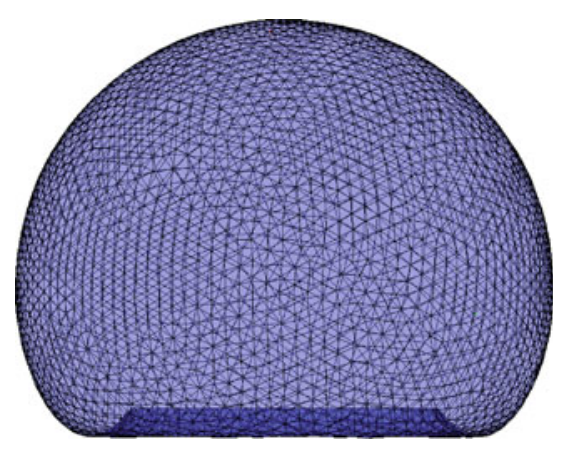

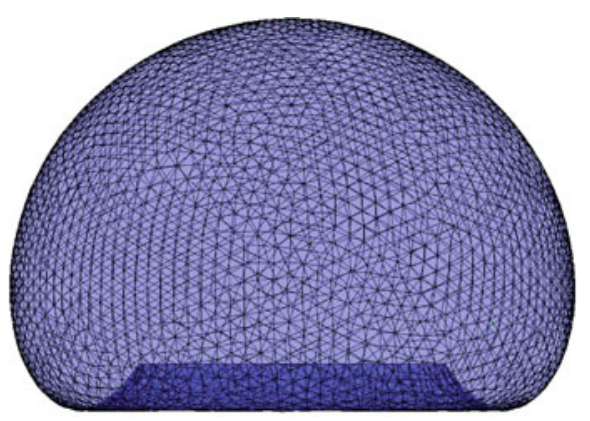

(d)

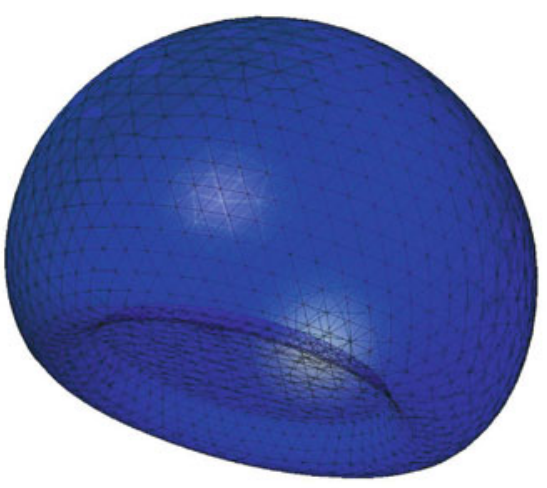

senting the quasi-incompressibility. This means that as long as the bulk modulus is high enough to prevent a large volume variation and ensures that the associated finite sound wave propagation speed is sensibly higher than the characteristic velocity of the problem, the approximation made can be accepted. Note that to obtain maximal performance, one must consider on one side that the bulk modulus must be high enough for the correct representation of the physics, but on the other hand it should be kept as small as possible to ensure the fast convergence of the iterative solvers.

\subsection{Deformation of an elastic plate subjected to water pressure}

Next example presents the deformation of an elastic plate subjected to water pressure. A water container of width $A=$ $0.1 \mathrm{~m}$ with water level $L=0.14 \mathrm{~m}$ is closed by an elastic cover of height $H=0.079 \mathrm{~m}$ and width $s=0.005 \mathrm{~m}$, which is fixed at the top (see Fig. 6). The cover is released and exposed to the water pressure, which induces deformation. The elastic cover is modeled with the following properties
- density $\rho=1,100 \mathrm{~kg} / \mathrm{m}^{3}$

- Young modulus $E=0.014 \mathrm{GPa}$

- Poisson ratio $v=0.4$

Results obtained with quasi-incompressible Updated Lagrangian fluid formulation are compared with the experimental ones reported in [36]. The comparison is shown in Fig. 7. Water was modeled with $\kappa=10^{7} \mathrm{~Pa}$. Snap shots are taken at time instances $t=0.04,0.08,0.12,0.16 \mathrm{~s}$.

\subsection{Deformation of an elastic membrane balloon filled with water}

Next, the results of a 3D simulation are presented. A truncated spherical shape membrane filled with water is fixed at its bottom and then is exposed to the water weight. The membrane of $0.1 \mathrm{~m}$ radius has a truncation base radius of 0.05 and $1.5 \mathrm{~mm}$ thickness. The material properties are summarized next:

- density $\rho=1,100 \mathrm{~kg} / \mathrm{m}^{3}$

- Young modulus $E=0.8 \mathrm{MPa}$

- Poisson ratio $v=0.2$ 


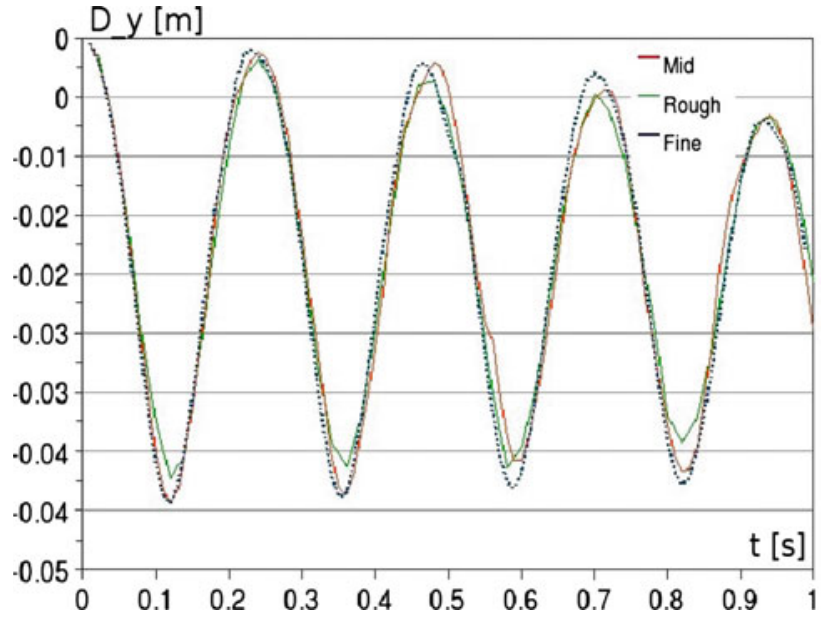

Fig. 9 Comparison of the vertical displacement $d_{y}$ for the membrane filled with water obtained with three different meshes

Results are presented for the water bulk modulus $\kappa=10^{6} \mathrm{~Pa}$. Figure 8 depicts the membrane at three time instances: at initial undeformed stage, maximum deformation and the final state.

Figure 9 presents the evolution of the vertical displacement $d_{y}$ of the upper-most point of the membrane obtained with three different meshes containing

1. 3,500 fluid linear tetrahedra and 1,200 membrane triangles

2. 8,000 fluid linear tetrahedra and 2,300 membrane triangles

3. 20,000 fluid linear tetrahedra and 4,000 membrane triangles

The rationale behind the choice of this example, is to assess the capability of the method to model a deforming object, while preserving the internal volume constraint. The motion of the membrane and its contact with the fluid is tracked automatically when the proposed approach is applied. This example does not require one to use very high values of the bulk modulus, as the velocities encountered are low. Since no free-surface is present, the volume variation is not influenced to any extent by the use of alpha-shape. As a consequence, the example shows the deviation of the proposed quasi-incompressible $\mathrm{FE}$ formulation from the ideally incompressible behavior, that theoretically should result in zero mass changes.

Table 8 shows maximal mass changes occurring for different values of bulk modulus. The relative volume variation is computed as following (density is assumed constant): $a_{\max }=\frac{\sum_{e l} V_{e l}^{n}}{V_{0}}$, where $V_{0}=\sum_{e l} V_{e l}(t=0)$ is the volume of the fluid in the initial state, and $V_{e l}^{n}$ is the volume the current step. The greatest volume loss and the volume loss at the equilibrium state are displayed.
Table 8 Maximal and average volume variation: Example 3.3

\begin{tabular}{lll}
\hline$\kappa$ & $a_{\max }(\%)$ & $a_{\text {end }}(\%)$ \\
\hline $10 \mathrm{E} 05$ & 2.43 & 1.6 \\
$10 \mathrm{E} 06$ & 0.26 & 0.14 \\
$10 \mathrm{E} 07$ & 0.015 & 0.01 \\
\hline
\end{tabular}

One can see that already for the bulk modulus of $K=1 \mathrm{MPa}$ the resulting mass loss is less than 0.5 percent. This clearly shows that the introduced slight compressibility does not bring in any considerable error as long as the volume conservation is concerned.

\section{Summary and conclusions}

The aim of this paper was to show that strongly coupled FSI problems can be efficiently solved by a monolithic method. A natural monolithic system was obtained by condensing pressure at the global level. The solution procedure was based on Newtons method, and the linearization procedure was presented. It was shown that the linearized pressure equation needs to be used only in the computation of the dynamic tangent stiffness. An "exact" pressure update equation was introduced. Pressure stabilization based upon the difference between consistent and lumped mass matrices assures pressure stability.

A matrix-free method for solving the linear system leads to a feasible method as it does not require explicit computation of the matrix resulting from the linearization of the gradient of pressure term.

It was shown that the value of the bulk modulus does not considerably affect the convergence rates of the non-linear procedure. Utilization of more sophisticated preconditioners can improve the efficiency of the method with respect to the convergence of an iterative solver. However already with the diagonally preconditioned CG the method is competitive for solution of the presented example problems for the range of bulk modulus up to $\approx 10 \mathrm{E} 7 \mathrm{~Pa}$. This value is far above the one that can be used in conjunction with classical penalty methods that use elemental pressure condensation and is sufficiently large for a correct representation of a wide range of physical phenomena. It is important to keep in mind that this limiting value of bulk modulus gives an indication rather than an actual limit.

Acknowledgments The support of the SEDUREC Project of the Consolider Programme of the Ministry of Science and Education of Spain is acknowledged. The authors also express their gratefulness to the Spanish Ministry of Science and Education for the support of the project XPRES (BIA2007-68120-C03-01). 
Table 9 Number of non-zero entries and respective matrix sizes

\begin{tabular}{lll}
\hline Parameter & $\mathbf{G M}_{p}^{-1} \mathbf{D}$ & $\mathbf{D}$ \\
\hline$n_{\text {non-zero per node in 2D }}$ & 18 & 6 \\
$n_{\text {non-zero per node in 3D }}$ & 124 & 26 \\
Size in 2D & $2 N \cdot 2 N$ & $N \cdot 2 N$ \\
Size in 3D & $3 N \cdot 3 N$ & $N \cdot 3 N$ \\
$n_{\text {non-zero total in 2D }}$ & $18 \cdot 2 N \cdot 2 N$ & $6 \cdot 2 N \cdot N$ \\
$n_{\text {non-zero total in 3D }}$ & $124 \cdot 3 N \cdot 3 N$ & $6 \cdot 3 N \cdot N$ \\
\hline
\end{tabular}

Table 10 Comparison of the number of the floating point operations

\begin{tabular}{llll}
\hline parameter & $\mathbf{G M}_{p} \mathbf{D}$ & Matrix free & speed-up \\
\hline nFLOPs in 2D & $72 \cdot N^{2}$ & $24 \cdot N^{2}+N$ & 3 \\
nFLOPs in 3D & $1116 \cdot N^{2}$ & $156 \cdot N^{2}+N$ & 7.2 \\
\hline
\end{tabular}

\section{Appendix 1}

Here the estimation is presented whose results were mentioned in Sect. 2.8. We shall compare the number of floating point operations, resulting when constructing the $\mathbf{G M}_{p}^{-1} \mathbf{D}$ matrix and when using the matrix-free method that involves only subsequent matrix-vector multiplications. Note that in both cases $\mathbf{M}_{p}$ is taken in the lumped format.

Table 9 summarizes the data necessary for the FLOPs estimation in both cases under the assumption of structured mesh. The number of non-zero entries $n_{\text {non-zero }}$ is estimated taking into account the structure of $\mathbf{G M}_{p}^{-1} \mathbf{D}$ and $\mathbf{D}$ matrices. In the former one each node contributes to the number of non-zero entries equal to the number of the neighbors of the neighbors of the node ("second neighbors") multiplied with the matrix size $(3 N * 3 N$ in $3 \mathrm{D}$ and $2 N * 2 N$ in $2 \mathrm{D})$. In the latter one, it is equal to the number of the nodal neighbors only, multiplied by the matrix size ( $3 N * N$ in $3 \mathrm{D}$ and $2 N * N$ in 2D). In the estimation of the total number of the non-zero entries, we do not consider the effect of the boundary nodes, which will slightly decrease the total number of non-zero entries. The total number of non-zero entries is therefore the product between the non zero contributions of a node and the matrix size, $N$ is the number of nodes in the discretization. Finally, Table 10 presents the cumulative FLOPS for either case. Note, that in the matrix free method we need to perform 3 steps of 2.8, where step 1 and step 3 lead to an equal number of FLOPS since $\mathbf{G}=\mathbf{D}^{T}$ and step 2 leads to N FLOPS, as $\mathbf{M}_{p}^{-1}$ is a lumped matrix of size $\mathbf{N}$.

The matrix-free procedure leads to a speed-up of $\approx 3$ in $2 \mathrm{D}$ and of $\approx 7.2$ in $3 \mathrm{D}$ in comparison with the direct construction of the $\mathbf{G M}_{p}^{-1} \mathbf{D}$ matrix.

\section{Appendix 2}

Time integration and linearization were presented in conjunction with the Backward Euler algorithm for the sake of clarity. However in the implementation of the present work a Newmark-Bossak scheme was used. The Newmark algorithm defines a second-order accurate unconditionally stable implicit time integration scheme. Bossak modification provides the improvement for highly geometrically non-linear problems, where classical Newmark method exhibits lack of stability. Bossak scheme introduces numerical dissipation, thus damping the high frequencies. We propose to use a Newmark-Bossak scheme for the time integration of fluid equations aiming at the monolithic coupling with structures. Newmark-Bossak scheme is widely used in the structural codes and we "adapt" the fluid formulation to fit the former one.

The Newmark-Bossak formulae for the acceleration and velocity read

$\overline{\mathbf{v}}_{n+1}=\frac{\overline{\mathbf{u}}_{n+1}-\overline{\mathbf{u}}_{n}}{\delta t}-\left(\frac{\gamma}{\beta}-1\right) \overline{\mathbf{v}}_{n}-\frac{\delta t}{2}\left(\frac{\gamma}{\beta}-2\right) \overline{\mathbf{a}}_{n}$

$\overline{\mathbf{a}}_{n+1}=\frac{\overline{\mathbf{u}}_{n+1}-\overline{\mathbf{u}}_{n}}{\beta \delta t^{2}}-\frac{1}{\beta \delta t} \overline{\mathbf{v}}_{n}-\left(\frac{1-2 \beta}{2 \beta}\right) \overline{\mathbf{a}}_{n}$

where $\gamma=\frac{1}{2}-\alpha^{B}$ and $\beta=\frac{\left(1-\alpha^{B}\right)^{2}}{4}$ with $\alpha^{B}>0$. If $\alpha^{B}=0$ we obtain the standard Newmark scheme at its optimal, without any numerical dissipation. Numerical dissipation control is obtained by varying the coefficient $\alpha^{B}$ [30]. Substituting Eq. (43) into Eq. (23) and subsequent linearization leads to the following tangent stiffness $\mathbf{H}_{B}$ and residual $\overline{\mathbf{r}}_{B}$.

$$
\begin{aligned}
\mathbf{H}_{B}= & \rho\left(1-\alpha^{B}\right) \mathbf{M} \frac{1}{\beta \delta t^{2}}-\mu \mathbf{L} \frac{\gamma}{\beta \delta t}-\rho c^{2} \mathbf{G} \mathbf{M}_{p}^{-1} \mathbf{D} \\
\overline{\mathbf{r}}_{B}= & \mathbf{F}_{n+1}+\mathbf{G} \bar{p}_{n+1}-\rho \mathbf{M}\left(\frac{\left(1-\alpha^{B}\right) \overline{\mathbf{u}}_{n+1}+\alpha^{B} \overline{\mathbf{u}}_{n}}{\beta \delta t^{2}}\right. \\
& \left.-\frac{\overline{\mathbf{v}}_{n}}{\beta \delta t}-\left(\frac{1-2 \beta}{2 \beta}\right) \overline{\mathbf{a}}_{n}\right) \\
& +\mu \mathbf{L}\left(\frac{\overline{\mathbf{u}}_{n+1}-\overline{\mathbf{u}}_{n}}{\delta t}-\left(\frac{\gamma}{\beta}-1\right) \overline{\mathbf{v}}_{n}\right)
\end{aligned}
$$

\section{References}

1. Küttler U, Wall W (2008) Fixed-point fluid-structure interaction solvers with dynamic relaxation. Computational Mechanics 43: $61-72$

2. Idelsohn SR, Del Pin F, Rossi R, Oñate E (2009) Fluid-structure interaction problems with strong added-mass effect. Int $\mathrm{J}$ Numer Methods Eng 80(10):1261-1294

3. Hughes TJR, Liu WK, Zimmermann TK (1981) LagrangianEulerian finite element formulation for incompressible viscous flows. CMAME 29:329-349 
4. Donea J, Huerta A (2003) Finite element method for flow problems. Wiley edition, New York

5. Rossi R, Oñate E (2010) Analysis of some partitioned algorithms for fluid-structure interaction. Eng Comput 27:20-56

6. Tezduyar TE, Behr M, Liou J (1992) A new strategy for finite element computations involving moving boundaries and interfaces - the deforming-spatial-domain/space-time procedure: I. the concept and the preliminary numerical tests. CMAME 94:339-351

7. Tezduyar TE, Behr M, Mittal S, Liou J (1992) A new strategy for finite element computations involving moving boundaries and interfaces - the deforming-spatial-domain/space-time procedure: II. computation of free-surface flows, two-liquid flows, and flows with drifting cylinders. CMAME 94:353-371

8. Mittal S, Tezduyar TE (1995) Parallel finite element simulation of 3D incompressible flows: fluid-structure interactions. IJNMF 21:933-953

9. Tezduyar TE, Sathe S, Keedy R, Stein K (2006) Space-time finite element techniques for computation of fluid-structure interactions. CMAME 195:2002-2027

10. Tezduyar TE, Sathe S (2007) Modeling of fluid-structure interactions with the space-time finite elements: solution techniques. IJNMF 54:855-900

11. Bazilevs Y, Calo VM, Hughes TJR, Zhang Y (2008) Isogeometric fluid-structure interaction: theory, algorithms, and computations. Comput Mech 43:3-37

12. Gerstenberger A, Wall W (2008) An extended finite element/ Lagrange multiplier based approach for fluid-structure interaction. Comput Methods Appl Mech Eng 197:1699-1714

13. Del Pin F, Idelsohn S, Oñate E, Aubry R (2007) The ALE/Lagrangian particle finite element method: a new approach to computation of free-surface flows and fluid object interactions. Comput Fluids $36: 27-38$

14. Legay A, Chessa J, Belytschko T (2006) An Eulerian-Lagrangian method for fluid-structure interaction based on level-sets. Int $\mathbf{J}$ Numer Methods Eng 195:2070-2087

15. Gerstenberger A, Wall W (2008) An extended finite element/ lagrange multiplier based approach for fluid-structure interaction. Comput Methods Appl Mech Eng 197:1699-1714

16. Osher SJ, Fedkiw RP (2006) Level set methods and dynamic implicit surfaces. Springer edition, Berlin

17. Belytschko T, Liu WK, Moran B (2003) Nonlinear finite elements for Continua and Structures. Wiley edition, New York

18. Oñate E, Idelsohn S, Del Pin F, Aubry R (2004) The particle finite element method: an overview. Int J Comput Methods 1:267-307

19. Idelsohn S, Oñate E, Del Pin F (2004) The particle finite element method: a powerful tool to solve incompressible flows with free-surfaces and breaking waves. Int J Numer Methods Eng 61: 964-989

20. Celigueta MA, Oñate E, Del Pin F, Idelsohn SR (2005) Possibilities of the particle finite element method (pfem) for hydrodynamic and fluid-structure interaction analysis of port structures. WIT Trans Built Environ 79:437-445
21. Idelsohn S, Marti J, Limache A, Oñate E (2008) Unified Lagrangian formulation for elastic solids and incompressible fluids. Application to fluid-structure interaction problems via the pfem. Comput Methods Appl Mech Eng 197:1762-1776

22. Larese A, Rossi R, Oñate E, Idelsohn SR (2008) Validation of the particle finite element method (pfem) for simulation of free surface flows. Eng Comput 25:385-425

23. Rossi R, Ryzhakov P, Oñate E (2009) A monolithic fe formulation for the analysis of membranes in fluids. Spatial Struct 24(4): 205-210

24. Oñate E, Idelsohn S, Celigueta M, Rossi R (2008) Advances in the particle finite element method for the analysis of fluid-multibody interaction and bed erosion in free surface flows. Comput Methods Appl Mech Eng 197

25. Idelsohn SR, Calvo N, Oñate E (2003) Polyhedrization of an arbitrary an arbitrary point set. J Comput Methods Appl Mech Eng 192:2649-2668

26. Akkiraju N, Edelsbrunner H, Facello M, Fu P, Mucke EP, Varela C (1995) Alpha shapes: definition and software. In: Proceedings of international computational geometry software workshop

27. Limache A, Idelsohn SR, Rossi R, Oñate E (2007) The violation of objectivity in Laplace formulations of the Navier-Stokes equations. Int J Numer Methods Fluid 54:639-664

28. Souza Neto EA, Peric D, Dutko M, Owen DRJ (1996) Design of simple low order finite elements for large strain analysis of nearly incompressible solids. Int J Solids Struct 33:3277-3296

29. Brezzi F, Bathe KJ (1990) A discourse on the stability conditions for mixed finite element formulations. Comput Methods Appl Mech Eng 82:27-57

30. Hughes T (2000) The finite element method. Linear static and dynamic FE analysis. Dover edition, New York

31. Gresho PM, Sani RL (1998) Incompressible flow and the finite element method. Wiley, New York

32. Zienkiewicz OS, Taylor RL, Nithiarasu P (2009) The finite element method for Fluid Dynamics, 6th edn, 3 volumes. Elsevier Butterworth Gheinemann edition, Amsterdam

33. Dadvand P, Rossi R, Oñate E, An object-oriented environment for developing finite element codes for multi-disciplinary applications. Arch Comput Methods Eng (to appear)

34. Hübner B, Walhorn E, Dinkler D (2004) A monolithic approach to fluid-structure interaction using space-time finite elements. Comput Methods Appl Mech Eng 193:23-26

35. Walhorn E, Kolke A, Hubner B, Dinkler D (2005) Fluid-structure coupling within monolithic model involving free surface flow. Comput Struct 83:2100-2111

36. Antoci C, Gallati M, Sibilla S (2007) Numerical simulation of fluid structure interaction by sph. J Comput Struct 85:879-890 